\title{
Cholinergic Control over Attention in Rats Prone to Attribute Incentive Salience to Reward Cues
}

\author{
Giovanna Paolone, Christopher C. Angelakos, Paul J. Meyer, Terry E. Robinson, and Martin Sarter \\ Department of Psychology and Neuroscience Program, University of Michigan, Ann Arbor, Michigan 48109-8862
}

\begin{abstract}
Some rats [sign-trackers (STs)] are especially prone to attribute incentive salience to reward cues, relative to others [goal-trackers (GTs)]. Thus, reward cues are more likely to promote maladaptive reward-seeking behavior in STs than GTs. Here, we asked whether STs and GTs differ on another trait that can contribute to poor restraint over behavior evoked by reward cues. We report that, relative to GTs, STs have poor control over attentional performance, due in part to insufficient cholinergic stimulation of cortical circuitry. We found that, relative to GTs, STs showed poor performance on a sustained attention task (SAT). Furthermore, their performance fluctuated rapidly between periods of good to near-chance performance. This finding was reproduced using a separate cohort of rats. As demonstrated earlier, performance on the SAT was associated with increases in extracellular levels of cortical acetylcholine (ACh); however, SAT performanceassociated increases in ACh levels were significantly attenuated in STs relative to GTs. Consistent with the view that the modulatory effects of ACh involve stimulation of $\alpha 4 \beta 2^{\star}$ nicotinic ACh receptors (nAChRs), systemic administration of the partial nAChR agonist ABT-089 improved SAT performance in STs and abolished the difference between SAT-associated ACh levels in STs and GTs. Neither the nonselective nAChR agonist nicotine nor the psychostimulant amphetamine improved SAT performance. These findings suggest that individuals who have a propensity to attribute high-incentive salience to reward cues also exhibit relatively poor attentional control. A combination of these traits may render individuals especially vulnerable to disorders, such as obesity and addiction.
\end{abstract}

\section{Introduction}

Research in both human and nonhuman animals suggests that a number of factors interact to confer vulnerability to developing addiction-like behaviors, including individual neuronal and behavioral vulnerabilities (e.g., structural abnormalities in cortical and striatal regions), impulsivity, maladaptive reward processing, and poor cognitive control (Rogers et al., 1999; Ersche et al., 2010, 2011, 2012; Winstanley et al., 2010; Goldstein and Volkow, 2011; Broos et al., 2012; Lucantonio et al., 2012; Robbins et al., 2012). For example, some rats are especially vulnerable to the lure of reward cues, due in part to variation in the extent to which individuals attribute incentive motivational properties (incentive salience) to reward cues (Flagel et al., 2009; Meyer et al., 2012). If a discrete cue, such as a lever, predicts delivery of a food reward, some rats [signtrackers (STs)] attribute incentive salience to the cue, as indicated by their propensity to approach it and work for it, whereas for others [goal-trackers (GTs)] the cue itself does not become an attractive and desirable incentive stimulus, upon lever-conditioned stimulus (CS) presentation they approach the food cup (Robinson and Flagel, 2009). Importantly, a dis-

Received Feb. 15, 2013; revised March 28, 2013; accepted April 2, 2013.

Author contributions: G.P., T.E.R., and M.S. designed research; G.P., C.C.A., and P.J.M. performed research; G.P. analyzed data; G.P., T.E.R., and M.S. wrote the paper.

This research was supported by PHS Grants 1P01 DA031656 and 1R01MH080332. We thank Abbott Laboratories for providing ABT-089. We thank Carillon Skrzynski for technical assistance. P.J.M. is now at SUNY Buffalo.

Correspondence should be addressed to Dr. Martin Sarter, University of Michigan, Department of Psychology, 530 Church Street, 4030 East Hall, Ann Arbor, Ml 48109-8862. E-mail: msarter@umich.edu.

DOI:10.1523/JNEUROSCI.0709-13.2013

Copyright $\odot 2013$ the authors $\quad 0270-6474 / 13 / 338321-15 \$ 15.00 / 0$ crete cocaine cue also acquires greater control over motivated behavior in STs than GTs (Saunders and Robinson, 2010). Dopamine signaling in the nucleus accumbens is necessary for mediating the attribution of incentive salience to Pavlovian reward cues in STs (Flagel et al., 2011; Saunders and Robinson, 2012), suggesting that mesolimbic processing of reward cues in STs differs fundamentally from GTs.

However, the ability to resist reward cues is not only a function of their incentive motivational properties, but also of the effectiveness of frontocortical attentional control processes. $\mathrm{Nu}$ merous studies have shown strong attentional biases toward food cues in obese people and to drug cues in addicts, but there is also considerable variation in the resulting consumption of attentional resources that have been hypothesized to contribute to strong cravings (for review, see Field and Cox, 2008). Indeed, reward cues will most likely goad individuals to action if a propensity to attribute incentive salience to drug cues is combined with relatively poor attentional control of such cues (for review, see Sarter and Paolone, 2011). Here we sought to explore the relationship between these two phenotypes by quantifying the attentional capacities of STs and GTs, and underlying activity of the prefrontal cholinergic input system, using a sustained attention task (SAT) (Demeter et al., 2008, 2011). This task was previously used in animals and humans to determine attentional control deficits (Demeter et al., 2013) and the mesolimbiccholinergic mechanisms that influence the strength of attentional control (Hasselmo and Sarter, 2011; St. Peters et al., 2011b; Sarter et al., 2006). The present results collectively indicate that ST is associated with relatively poor attentional control and attenuated levels of performance-associated cortical acetylcholine (ACh) re- 
lease, and that stimulation of $\alpha 4 \beta 2^{\star}$ nAChRs improves the attentional performance in STs.

\section{Materials and Methods}

Subjects. An initial experiment ("cohort 1"; $N=324$ ) was conducted using Sprague Dawley rats (250-300 g) purchased from Harlan $(n=$ $132)$ and from Charles River $(n=192)$. To assess the reliability of the differences between STs and GTs on SAT performance, a completely separate cohort ("cohort 2"; $N=153$ ) were screened from rats obtained again from Harlan $(n=128)$ and Charles River $(n=25)$. Across the two cohorts, a total of $N=477$ rats was screened which yielded 182 STs and 170 GTs ( 32.5 and $35.6 \%$ of the total population, respectively). Fifty-two STs and 46 GTs were randomly selected for training and testing in the SAT; the rest were either not tested or used in other experiments. Because prior studies indicated that neither the development of Pavlovian conditioned approach behavior nor sustained attentional performance differ between the sexes and levels of circulating estrogens (McGaughy and Sarter, 1999; K. Pitchers, M. Sarter, and T.E. Robinson, unpublished observations), these experiments were conducted with male rats.

Rats were housed in pairs and kept on a reverse light-dark cycle (lights off at 8:00 A.M.) and handled daily for at least 1 week before screening in the Pavlovian approach test. After completion of the screening procedure, animals were individually housed in a temperature $\left(23^{\circ} \mathrm{C}\right)$ and humidity-controlled (45\%) environment with a $12 \mathrm{~h}$ light/dark cycle (lights on at 7:00 A.M.). Animals were handled extensively before the beginning of SAT training, and were water-deprived by restricting access to a 10 min period after each operant training or practice session. Water was also provided as a reward during task performance (see below). On days not tested, water access was increased to a total duration of $30 \mathrm{~min}$. Food (Rodent Chow, Harlan Teklad) was available ad libitum. All procedures were conducted in adherence with protocols approved by the University Committee on Use and Care of Animals at the University of Michigan and in AAALAC (Association for Assessment and Accreditation of Laboratory Animal Care)-accredited laboratories.

Use of rats from cohorts 1 and 2, and timeline of experiments. Rats were tested in two cohorts to test the reliability of the SAT performance difference between STs and GTs. Rats from cohort 2 were screened for Pavlovian Conditioned Approach (PCA) behavior $\sim 1$ year after the PCA screening of cohort 1 . Rats of both cohorts reached the final stage of the SAT after 18 (cohort 1 ) and 17 sessions (cohort 2).

Different subgroups of cohort 1 were used for the in-depth analysis of SAT performance (data from sessions 58-60; this subgroup was not tethered, not tested in special microdialysis chambers, and not administered drugs), microdialysis during SAT performance, and for pharmacological tests. The administration of the three drugs, doses, and vehicle was counterbalanced over individual rats, and took place across sessions 30 105 (ABT-089), 30-109 (amphetamine), and 43-93 (nicotine). Microdialysis experiments were conducted across task sessions 30-75. A portion of animals of cohort 2 underwent microdialysis during SAT performance to confirm the attenuated extracellular cortical ACh levels in STs relative to GTs.

PCA screening. The extent to which behavior was lever or food cupdirected was quantified using a PCA index score (below). Pavlovian training procedures were similar to those previously described (a metaanalysis of PCA screening in $>1800$ rats) (Meyer et al., 2012).

Apparatus and procedures. On $2 \mathrm{~d}$ preceding testing, rats were given $\sim 25$ banana-flavored sucrose pellets ( $45 \mathrm{mg}$, BioServ) in their home cages. Rats were tested in conditioning chambers (MedAssociates; $20.5 \times$ $24.1 \mathrm{~cm}$ floor area, $29.2 \mathrm{~cm}$ high). A food magazine, into which an automatic feeder delivered sucrose pellets, was located in the center of one side of the cage. Infrared photobeam breaks detected magazine entries. On either the left or right side of the magazine was a retractable lever with a LED backlight that was illuminated only when the lever was inserted into the chamber. Lever deflections were used to record lever contacts. A red house light was located near the ceiling of the opposite side of the chamber; its illumination signaled the beginning of the test sessions.

On the first day of testing ("pretraining"), rats were placed into transport boxes, transferred to the testing room, and placed into the conditioning chambers. The house light was illuminated after a $5 \mathrm{~min}$ habituation period. Twenty-five sucrose pellets were then delivered on a VI-30 (0-60 s) schedule. The lever was retracted throughout this session, which lasted, on average, $12.5 \mathrm{~min}$. Rats consumed all the pellets during this and subsequent PCA sessions. For the five daily PCA sessions, the house light signaled the session start after $1 \mathrm{~min}$, and then rats were presented with 25 lever-pellet pairings delivered on a VI-90 (30-150 s) schedule. The CS for each trial was the extension of the illuminated lever into the chamber for $8 \mathrm{~s}$. A sucrose pellet was delivered into the magazine upon retraction of the lever. These sessions lasted, on average, $37.5 \mathrm{~min}$.

PCA measures, classification criteria, and statistical analysis. The two main variables of interest were lever presses and magazine entries during the CS periods.

$P C A$ index. From the lever-press and magazine-entry data, we calculated three measures of approach. (1) Response bias was defined as the difference between lever presses and magazine entries during the CS, expressed as a proportion of the total responses [(lever presses - magazine entries)/(lever presses + magazine entries)]. (2) Latency score was calculated as the difference between the latencies to approach the lever and the magazine upon CS presentation; this difference was normalized by dividing by the maximum $8 \mathrm{~s}$ latency [(magazine latency - lever latency)/8]. (3) Probability difference was calculated as the difference between the probabilities of pressing the lever during the CS (i.e., number of trials with lever press/25 trials) minus the probability of entering the magazine. Thus, PCA index score consisted of (probability difference score + response bias score + latency difference score/3). This formula produces values ranging from -1.0 to +1.0 . A score of +1 means approach to and contacting the lever on every trial, -1 indicates magazine entry on every trial, and a score of 0 that lever contacts and magazine entries were equally distributed across trials.

The PCA index scores obtained on day 5 were used to classify rats as STs or GTs. Rats were considered STs if they obtained scores ranging from +0.4 to +1.0 , indicating that lever-directed behavior was more than twice as frequent as food cup-directed behavior. Rats with scores ranging between -0.4 and -1.0 were classified as GTs (see Results for actual scores of rats that were randomly selected from screened groups for the present experiments). Rats with intermediate scores were not used in these studies. Approach responses (response probability, number of contacts, and latency) were analyzed with repeated-measures ANOVA with phenotype (STs, GTs) as the between groups measure and training day (1-5).

\section{SAT performance}

Behavioral apparatus, training, and testing procedures. Behavioral training and testing took place using 12 operant chambers located inside sound attenuating chambers (MedAssociates). The chambers were equipped with an intelligence panel consisting of three panel lights $(2.8 \mathrm{~W})$, two retractable levers, and a water dispenser $(40-45 \mu$ l of water per delivery). A house light $(2.8 \mathrm{~W})$ was located on the rear wall. These boxes featured a taller recessed water delivery area $(9.0 \times 5.0 \mathrm{~cm}$, height $\times$ width $)$ to allow cannulated animals to drink, and an opening in the ceiling of the operant chamber and sound attenuating chamber to allow for dialysis lines and pumps to remain outside the chambers. Signal presentation, lever operation, reinforcement delivery, and data collection were controlled by a Pentium PC and Med-PC for Windows software (v 4.1.3; Med-Associates).

Animals were initially trained to lever-press using a modified fixed ratio- 1 schedule for water reinforcement. After at least three consecutive sessions per day of $>120$ reinforced lever presses, animals entered the first stage of SAT training. Thereafter animals were trained to discriminate between a signal ( $1 \mathrm{~s}$ illumination of the central panel light) and a nonsignal (no illumination) event. Two seconds after such events, the levers were extended into the chamber. On signal trials, a response on the left lever was reinforced and termed a "hit," whereas a response on the right lever was not reinforced and termed a "miss". On nonsignal trials, a response on the right lever was reinforced and termed a "correct rejection," whereas a response on the left lever was not reinforced and termed a "false alarm". Half of the animals were trained using the reverse set of rules. If no response occurred within $4 \mathrm{~s}$, the levers were retracted and an omission was recorded. Signal and nonsignal events were pre- 
sented in pseudorandom order for 81 trials each (total of 162 trials) per session. The intertrial interval (ITI) was $12 \pm 3$ s. During this stage of training, incorrect responses were followed by up to three correction trials in which the previous trial (signal or nonsignal) was repeated. In the event of an incorrect response on the third correction trial, a forced trial was initiated in which only the correct lever was extended for $90 \mathrm{~s}$ or until the animal made a response. When the forced trial was a signal trial, the central panel light remained illuminated while the left lever was extended. The house light was not illuminated during this training stage. After at least five consecutive days of stable performance, defined as $>70 \%$ hits and $>70 \%$ correct rejections, multiple signal durations (500, $50,25 \mathrm{~ms}$ ) were introduced. Trial type and signal duration continued to be pseudo-randomly determined for each trial. Session length was set at $64 \mathrm{~min}$ to allow for post hoc analysis of performance over 8 min blocks of trials. The pseudo-random selection of trial type (signal versus nonsignal) and signal duration was designed to ensure that approximately half of the trials per block were signal trials, and that equivalent numbers of 500,50 , and $25 \mathrm{~ms}$ signals were presented during each block. At this point, correction trials and forced trials were discontinued, and the event rate was increased by reducing the ITI to $9 \pm 3 \mathrm{~s}$. After at least $7 \mathrm{~d}$ of stable performance, defined by at least $70 \%$ hits to $500 \mathrm{~ms}$ signals, $70 \%$ correct rejections, and $<20 \%$ omissions, animals began training in the final version of the task. The final version was identical to the previous training stage except that the house light was illuminated throughout the session. This final step is essential for assessing sustained attention performance, as it requires the animals to constrain their behavior and maintain persistent attention to the intelligence panel. Once animals reached this final stage of training, they remained on task for an additional 75 sessions.

Behavioral measures and data analysis. The evidence in support of the validity of performance measures, in terms of indicating sustained attention performance, in humans (Demeter et al., 2008), rats (McGaughy and Sarter, 1995; Demeter et al., 2008) and mice (St. Peters et al., 2011a) was previously reported. For each session, hits, misses, correct rejections, false alarms, and omissions were recorded. The relative number of hits (hits/hits + misses) was calculated for each signal length, and the relative number of correct rejections (correct rejections/correct rejections + false alarms) was also calculated. As an overall measure of attentional performance that integrates both the relative number of hits $(h)$ and the relative number of false alarms $(f)$, an overall performance score (SAT score) was calculated in accordance to: SAT score $=(h-f) /[2(h+f)-(h+$ $f)^{2}$. This index was derived from the sensitivity index (Frey and Colliver, 1973), except that the SAT score is based on the relative number of hits and false alarms, as opposed to the probabilities for hits and false alarms, and thus is not confounded by errors of omission. SAT scores range from +1.0 to -1.0 , with +1.0 indicating that all responses were hits and correct rejections, 0 indicating an inability to discriminate between signal and nonsignal events, and -1.0 indicating that all responses were misses and false alarms. SAT scores were calculated for each signal duration $\left(\mathrm{SAT}_{500,50,25}\right)$ or averaged over all durations. Errors of omission were recorded separately. Performance measures were calculated for each of the eight task blocks. To characterize performance levels over the $75 \mathrm{~d}$ of SAT performance, these data were blocked in five blocks of $15 \mathrm{~d}$ each and measures of performance during the last $3 \mathrm{~d}$ of each block were averaged for each rat and used for this analysis. As the performance of GTs and STs did not further improve after 45 sessions, the comparisons between cohorts 1 and 2 were based on the performance during sessions 43-45, whereas the more detailed analysis of SAT performance in STs and GTs was based on data from sessions 58-60 of the animals of cohort 1 .

Good, intermediate, and poor performance. We observed that SAT performance in STs fluctuated considerably across a test session. Furthermore, we observed that blocking performance in 3 min blocks, consisting of $\sim 15$ trials) effectively captured these fluctuations. To analyze these fluctuations in STs and GTs, we categorized SAT scores, averaged over 3 min blocks of trials, as indicating "good," "intermediate," or "poor" performance. As SAT scores $>0.17$ indicate above chance-level performance ( $>58 \%$ hits and $>58 \%$ correct rejections), SAT scores (averaged over signal duration) lower than 0.17 were classified as "poor," SAT scores $>3 \times$ chance-level were classified as "good," and performance in between as "intermediate" (see Fig. 4a). We used these categorizations to analyze effects of phenotype and drug on the number of 3 min periods at each performance level as well as the duration of performing uninterrupted at each level in a session.

Video scoring of SAT sessions. SAT sessions performed by a subset of STs and GTs ( $n=6$ per each phenotype; cohort 1$)$ were video taped for off-line analyses (CVC-130R, Speco Technologies; resolution, 420 TV lines). Off-line analyses were performed by undergraduate research assistants blinded to the phenotypes. These analyses were guided by the hypothesis that STs' performance may have been characterized by impulsive responding (Lovic et al., 2011), which in the SAT, may cause a propensity to respond to the proximal lever on lever extension, rather than (if required given the animal's position and the location of the correct lever) withholding responding and switching to the correct opposite lever. Therefore, these analyses focused on the position of the animals before a signal or nonsignal event. Specifically, we determined the proportion of trials during which rats were positioned, $1 \mathrm{~s}$ before a signal or, in the case of nonsignal trials, before lever extension, (1) in front of the hit/false alarms versus (2) miss/correct rejection lever, (3) in front of the water port (mouth in the water port opening), or (4) away from the intelligence panel (forepaws and head positioned in the contralateral half of the chamber). Furthermore, as we wished to gain more insight into potential overt behavioral correlates of shifts from good to poor performance, this analysis was based, per rat, on 30 trials constituting an abrupt and complete shift from good (the last 15 trials performing at the good level) to poor performance (the first 15 trials performing at the poor level, as defined above).

\section{Microdialysis during SAT performance}

Implantation of guide cannula. After reaching stable SAT scores $(<20 \%$ variation over three consecutive sessions), a subset of animals underwent stereotaxic surgery to implant a guide cannula in right medial prefrontal cortex (mPFC). This region was selected because of prior evidence, from rats and mice, indicating SAT-associated increases in cholinergic neurotransmission occur selectively in that region (Apparsundaram et al., 2005; Parikh et al., 2013). Surgery was performed under aseptic conditions. Animals were anesthetized initially with $4-5 \%$ isoflurane in an anesthetic chamber (Anesco/Surgivet). Gas was carried via oxygen at a flow rate of $0.6 \mathrm{~L} / \mathrm{min}$. Animals' heads were shaved using electric clippers and cleaned with an alcohol wipe. Animals were then mounted to a stereotaxic instrument (David Kopf Instruments). Ophthalmic ointment was used to lubricate animals' eyes. Isoflurane was administered via a facemask and the levels were adjusted to $1.5-2 \%$ for the remainder of surgery. Microdialysis guide cannulas (Model MAB 4.15.IC, Microbiotech) were implanted above the $\mathrm{mPFC}$ using the coordinates measured from bregma: AP, $2.9 \mathrm{~mm}$; ML, $0.6 \mathrm{~mm}$; DV, $0.6 \mathrm{~mm}$ below dura. To prevent clogging, the guide cannulas were equipped with stainless steel stylets. A headstage to hold the guide cannula in place was constructed using surgical screws implanted into the skull and dental cement. Animals were given injections of an antibiotic (amikacin, $250 \mathrm{mg} / \mathrm{ml}, 0.1 \mathrm{ml}$, i.p.) and an analgesic (buprenorphine, $0.01 \mathrm{mg} / \mathrm{kg} / \mathrm{ml}$, i.p.). The area around the headstage was coated with neosporin. Following surgery, animals were returned to their home cages and given ad libitum access to food and water for $4 \mathrm{~d}$. Thereafter the water deprivation schedule and SAT training was reinitiated. After animals regained stable presurgery performance levels for at least $3 \mathrm{~d}$, tubing was attached to the stylets of the guide cannulas to habituate the animals to performing under microdialysis conditions.

Microdialysis sessions. Microdialysis sessions began with the removal of the stylet and the insertion of a removable concentric probe with a 3.0 $\mathrm{mm}$ membrane tip (Model MAB 4.15.3; membrane o.d. $0.2 \mathrm{~mm}$, shaft o.d. $0.18 \mathrm{~mm}$; Microbiotech) into the PFC. Animals were perfused at a rate of $2.0 \mu \mathrm{l} / \mathrm{min}$ with artificial CSF (aCSF), $\mathrm{pH} 6.8 \pm 0.1$, containing the following (in mM): $126.5 \mathrm{NaCl}, 27.5 \mathrm{NaHCO}_{3}, 2.4 \mathrm{KCl}, 0.5 \mathrm{NA}_{2} \mathrm{SO}_{4}, 0.5$ $\mathrm{KH}_{2} \mathrm{PO}_{4}, 1.2 \mathrm{CaCl}_{2}, 0.8 \mathrm{MgCl}_{2}$, and 5.0 dextrose. Animals were placed into the operant chambers and the probes were perfused for at least 120 min to allow ACh efflux to stabilize. After this period, collection of baseline samples began, with dialysates collected every $8 \mathrm{~min}$. The last three collections before task onset were averaged to calculate basal ACh efflux. Animals received sterile saline ( $1 \mathrm{ml} / \mathrm{kg}$; i.p.) or ABT-089 $(0.02 \mathrm{mg} / \mathrm{kg}$; 
i.p.) 20 min before task onset (see below). Following baseline collections, the task began and eight SAT-associated collections were obtained. Five additional samples were collected posttask. Animals were then removed from the operant chambers, the microdialysis probes removed, the stylets reinserted, and rats were returned to their home cages.

Determination of ACh concentrations. Dialysate samples were stored at $-80^{\circ} \mathrm{C}$ until they were analyzed by high performance liquid chromatography with electrochemical detection (ESA). The mobile phase used contained $35 \mathrm{~mm}$ sodium phosphate, $0.43 \mathrm{~mm}$ ethylenediamine tetracetic acid tetrasodium salt, and $5 \mathrm{ml} / \mathrm{L}$ ProClin (BASi). ACh was separated from choline on a $250 \mathrm{~mm}$ analytical column and catalyzed on a postcolumn solid-phase reactor containing acetylcholinesterase and choline oxidase. ACh was then hydrolyzed to acetate and choline, and choline oxidized to hydrogen peroxide and betaine. The amount of hydrogen peroxide corresponding to ACh was then detected using a "peroxidasewired " glassy carbon electrode with an applied potential of $-200 \mathrm{mV}$. To calculate the concentration of ACh in each sample, the integral of the area under the peak was taken and fit to a regression line containing known values of $\mathrm{ACh}$ in the expected range of the in vivo dialysates. The in vitro recovery of probes varied from 8 to $15 \%$. The detection limit of this system averaged $2.0 \mathrm{fmol} / 15 \mu \mathrm{l}$. Following completion of the experiments, animals were given an overdose of sodium pentobarbital and perfused with phosphate buffer solution and then formalin (Fisher). Brains were postfixed in formalin until the next day. The brains were then transferred into a $30 \%$ sucrose phosphate-buffer solution and allowed to sink. A microtome was used to slice the brains into 50- $\mu$ m-thick sections, which were kept in phosphate-buffer solution. The sections surrounding and including the probe site were mounted onto gelatin-coated glass slides and allowed to dry completely before being stained with a cresyl violet. Data from rats with placements with less than half of the length of the $3 \mathrm{~mm}$ active membrane situated within the prelimbic region were rejected from final analysis (leading to the rejection of data from two rats).

\section{Effects of ABT-089, nicotine, and amphetamine on SAT}

performance and SAT-associated ACh release

The two doses per compound and vehicle were administered using a counterbalanced regimen. Drugs were prepared in $1.0 \mathrm{ml} / \mathrm{kg} 0.9 \%$ sterile saline. Drugs and vehicle were administered intraperitoneally and given 20 min before the onset of the SAT session, with animals placed in the test chambers during this period. Consecutive doses were separated by a minimum of 3 days/sessions or as many sessions required for the animals to regain stable predrug performance levels. Tests of the effects of the different drugs were separated by $8-10 \mathrm{~d}$ washout periods during which animals continued daily SAT practice. Group sizes for assessing drug effects were $n=12$ for both GTs and STs. Approximately half of the animals received all three drugs and doses, and approximately one-third were treated with only 2 or 1 compound.

Subgroups of animals from cohort 1 were assigned to be treated with ABT-089, nicotine, and or D-amphetamine. ABT-089 [2-methyl-3-(2(S)-pyrrolindinylmethoxy) pyridine dihydrochloride salt; batch no. 318857-1; MW, 265.18) was provided by Abbott Laboratories. ABT-089 is a partial agonist showing selectivity for the cytisine-binding site on the $\alpha 4 \beta 2^{\star}$ subtypes of the nicotinic acetylcholine receptor (Decker et al., 1997; Sullivan et al., 1997; Rueter et al., 2004; nAChR). The effects of this compound were assessed in STs and GTs (STs, $n=12$; GTs, $n=13$; vehicle, $0.02,0.10 \mathrm{mg} / \mathrm{kg}$; based on salt weight), guided by the hypothesis that stimulation of $\alpha 4 \beta 2^{\star}$ nAChRs benefits attentional control (Sarter and Paolone, 2011), in part by augmenting the mPFC mechanisms that mediate the detection of cues in attentional contexts (Parikh et al., 2007, 2008, 2010; Howe et al., 2010). Doses were based on pilot data showing enhancement of SAT performance by ABT-089 in unscreened rats and in the presence of a distractor (W.M. Howe and M. Sarter, unpublished observations).

Administration of nicotine has been frequently reported to benefit the attentional performance of humans and laboratory animals (Hahn et al., 2003, 2007; Newhouse et al., 2011). Although our own studies indicated that the effects of nicotine are not robust unless its effects at the $\alpha 7$ nAChR are blocked (Howe et al., 2010), the paucity of knowledge about the status of nAChRs in STs motivated us to test the effects of this nonselective nAChR agonist nicotine hydrogen tartrate (Sigma-Aldrich) which was administered to STs and GTs $(n=11$ each $)$ at the lowest and highest dose ( 0.02 and $0.40 \mathrm{mg} / \mathrm{kg}$, base weights) tested in our prior study in SAT performing rats (Howe et al., 2010).

Previous studies in unscreened rats did not indicate amphetamineinduced enhancement of SAT performance (McGaughy and Sarter, 1995) but these findings do not exclude the possibility that STs rats with lower levels of performance (below) benefit from psychostimulant treatment. Therefore, we tested the effects of a psychostimulant compound, D-amphetamine sulfate (Sigma-Aldrich; $0.25,0.75 \mathrm{mg} / \mathrm{kg}$ based on salt weight) in 11 animals of each phenotype.

Statistical methods and major independent and dependent variables. In all experiments, the phenotype of animals (STs vs GTs) was the major independent, between-subject variable. Additional independent variables were task parameters [block of trials, signal-duration (where applicable) and dose of drug (all within-subject)]. The primary dependent measure of SAT performance was the overall performance level as indicated by the SAT score (see below). Secondary analyses concerned the individual measures of response accuracy in signal and nonsignal trials (hits and correct rejections, respectively). Note that misses and false alarms are the inverse of hits and correct rejections, respectively, and thus were not analyzed. Errors of omission were separately analyzed and did not enter the calculation of SAT scores. For the experiments involving microdialysis, percentage change from baseline extracellular ACh levels was the main dependent variable.

Because of the complexity of the designs used for the multiple experiments described below, the main factors and statistical methods are identified briefly in the Results section. Generally, mixed-design ANOVAs were used to determine the effects of group (STs, GTs; between-subject factor), task parameter [signal duration (where applicable) and block] and dose (within-subject factors). Post hoc multiplecomparisons were conducted using $t$ test and Fisher's LSD test. Statistical analyses were performed using SPSS for Windows (V. 17.0; SPSS). In cases of violation of the sphericity assumption Huyhn-Feldt corrected $F$ values are given. Alpha was set at 0.05 or, where required (see Results), at fractions of that. Exact $p$ values are reported for significant results, as recommended earlier (Greenwald et al., 1996).

\section{Results}

\section{STs/GTs screening of cohorts 1 and 2}

For cohort 1 , of $n=324$ rats, 37\% were classified as STs and $38.3 \%$ as GTs. Of these rats, 22 STs and 18 GTs were randomly selected for SAT testing; the remaining rats were used for other experiments. These STs and GTs rats had PCA index scores between 0.54 and $0.96(\mathrm{M}$; SEM, $0.79 \pm 0.02)$ and between -0.65 and -0.94 (M; SEM: $0.84 \pm 0.02)$, respectively. STs and GTs increasingly approached the lever or magazine, respectively, as indicated by changes in the probability, number of contacts, and approach latency over sessions. This was supported statistically by a significant interaction between the effects of phenotype (STs, GTs) and session ( $1-5$, within-subject factor) for all six measures $\left(\alpha=0.05 / 6 ; F_{(4140)}>20.00, p<0.00001\right.$; note that because of missing data from 3 animals for days 1 and 2, this mixed model ANOVA was based on $N=37$ ).

For cohort 2, of $n=153$ rats, $40.5 \%$ were classified as STs and $30.1 \%$ as GTs. Of these rats, 30 STs and 28 GTs were randomly selected for further testing. These STs and GTs rats had PCA index scores between 0.45 and 0.92 (M; SEM, $0.74 \pm 0.02$ ) and between -0.51 and -0.97 (M; SEM, $-0.80 \pm 0.02)$, respectively. Similar to cohort 1 , STs and GTs increasingly approached the lever or magazine respectively (phenotype $\times$ session; $\alpha=0.05 / 6$; $F_{(4220)}>41.00, p<0.00001$; because of one missing data point this analysis was based on $N=57$ ).

PCA behavior of the two cohorts did not differ significantly, as indicated by the absence of a main effect of cohort on day 5 index 


\section{Lever (CS) directed behavior "Sign-Tracking"}
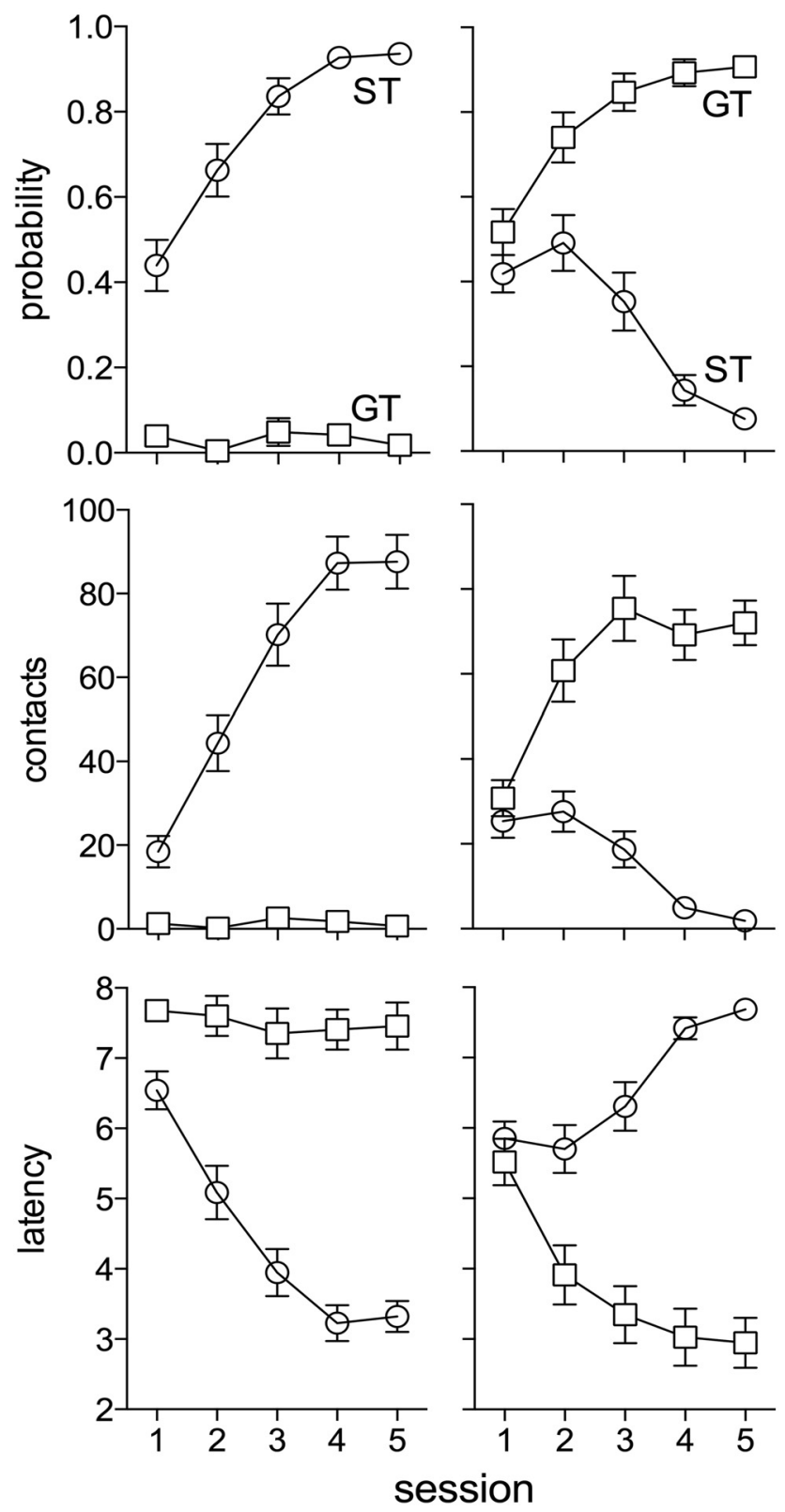

Figure 1. PCA performance by STs and GTs. Lever-directed (left column) or food portdirected (right column) behavior (probability, number of contacts, latency; M, SEM) over $5 \mathrm{~d}$ (or sessions) of Pavlovian training (see Materials and Methods for detailed description of measures). These measures were used to calculate the PCA index (see Materials and Methods). The data shown are collapsed across cohorts 1 and 2 (STs: $n=52 ; G T s: n=46$ ).

scores $\left(F_{(1,94)}=0.17, p=0.67\right)$. Figure 1 represents the PCA screening data for sessions $1-5$ for rats from both cohorts combined.

\section{SAT performance: cohort 1}

Acquisition and asymptotic performance

As rats from cohort 1 were assigned to parallel experiments on SAT-associated ACh levels and the effects of drugs (below), this analysis was based on subgroups of rats ( $n=12$ per phenotype) that were neither tethered and tested in special chambers for microdialysis nor treated with drugs and which were kept on the SAT for 75 sessions. STs and GTs advanced through the various stages of task training (see Materials and Methods) and did not differ at the rate with which they reached the final version of the SAT (number of sessions to final version of the task: STs, $19.9 \pm$ 2.82 ; GTs, $\left.15.80 \pm 1.81 ; t_{(22)}=1.48, p=0.15\right)$. As illustrated in Figure $2 a$, depicting the overall measure of performance, the SAT score, STs and GTs improved their performance until reaching asymptotic levels of SAT performance. However, the performance of STs remained below that of GTs across the entire period on the SAT. Mixed-model ANOVA of the effects of phenotype (STs versus GTs) on SAT scores over 5 blocks of sessions (withinsubject factor) confirmed that STs performed at relatively lower levels than GTs (main effect of phenotype; $F_{(1,23)}=19.37, p<$ 0.001 ) and that both groups' performance improved in parallel (main effect of block: $F_{(4,92)}=16.30, p<0.001$; interaction: $\left.F_{(4,92)}=0.74, p=0.56\right)$. One-way post hoc ANOVAs on the effects of blocks of sessions confirmed that both groups of animals improved their performance over sessions 15-75 (STs: $F_{(4,24)}=6.37, p<0.001$; GTs: $\left.F_{(4,22)}=11.96, p<0.001\right)$ (Figure $2 a$ shows multiple-comparisons).

A complete description of the performance of STs and GTs on the SAT is illustrated in Figures $2 b-e$ (based on data from sessions $58-60)$. In terms of overall performance, as indicated by the SAT score, a mixed model ANOVA on the effects of phenotype, signal duration and $8 \mathrm{~min}$ blocks of trials (within-subject factors) reproduced the difference in performance between STs and GTs (main effect of phenotype: $F_{(1,22)}=19.91, p<0.001$ ) and also indicated a main effect of signal duration $\left(F_{(2,44)}=220.93, p<\right.$ 0.001 ) but no effects of block (eight 8 min blocks of trials) and no interactions involving phenotype (all $F<1.44$, all $p>0.14$ ) (Fig. 2b).

The analysis of the individual measures of response accuracy in signal (hits) and nonsignal trials (correct rejections) indicated that the relatively lower level of performance by STs largely reflected their relatively lower hit rate (main effect of phenotype: $F_{(1,22)}=13.84, p=0.001$ ) (Fig. $2 c$ ). (Note that misses and false alarms are the inverse of hits and correct rejections, respectively, and thus were not analyzed.) As expected, hits were signal duration-dependent $\left(F_{(2,44)}=181.32, p<0.001\right)$ but the effect of phenotype was not moderated by signal duration or block (all interaction involving phenotype: all $F<2.63$, all $p>0.11$ ).

Further supporting lower hits as the main source for lower SAT performance in STs, the relative number of correct rejections did not differ between STs and GTs (main effect of phenotype: $F_{(1,22)}=0.03, p=0.87$ ) (Fig. $2 d$ ). However, all animals scored more correct rejections during early when compared with later blocks of trials (block: $F_{(7154)}=2.48, p=$ 0.03 ; phenotype $\times$ block: $F_{(7154)}=1.80, p=0.10$; multiplecomparisons, using Fisher's LSD test, indicated that correct rejections during blocks 5-8 were significantly lower than during block 3 , and correct rejections during block 8 were significantly lower than during block 4).

Errors of omission did not differ between STs and GTs. They omitted $<15 \%$ of trials throughout the session (main effect of phenotype: $F_{(1,22)}=0.72, p=0.41$ ) (Fig. $2 e$ ), All rats omitted more trials toward later blocks of trials [main effect of block of trials (within-subject factor): $F_{(7154)}=10.75, p<0.001$; phenotype $\times$ block $F_{(7154)}=2.32, p=0.09$; multiple-comparisons, using Fisher's LSD test, indicated that omissions were significantly higher during blocks $4-8$ than during block 2, during block 7 than blocks $1-6$, and higher during block 8 than during blocks 1-7]. 

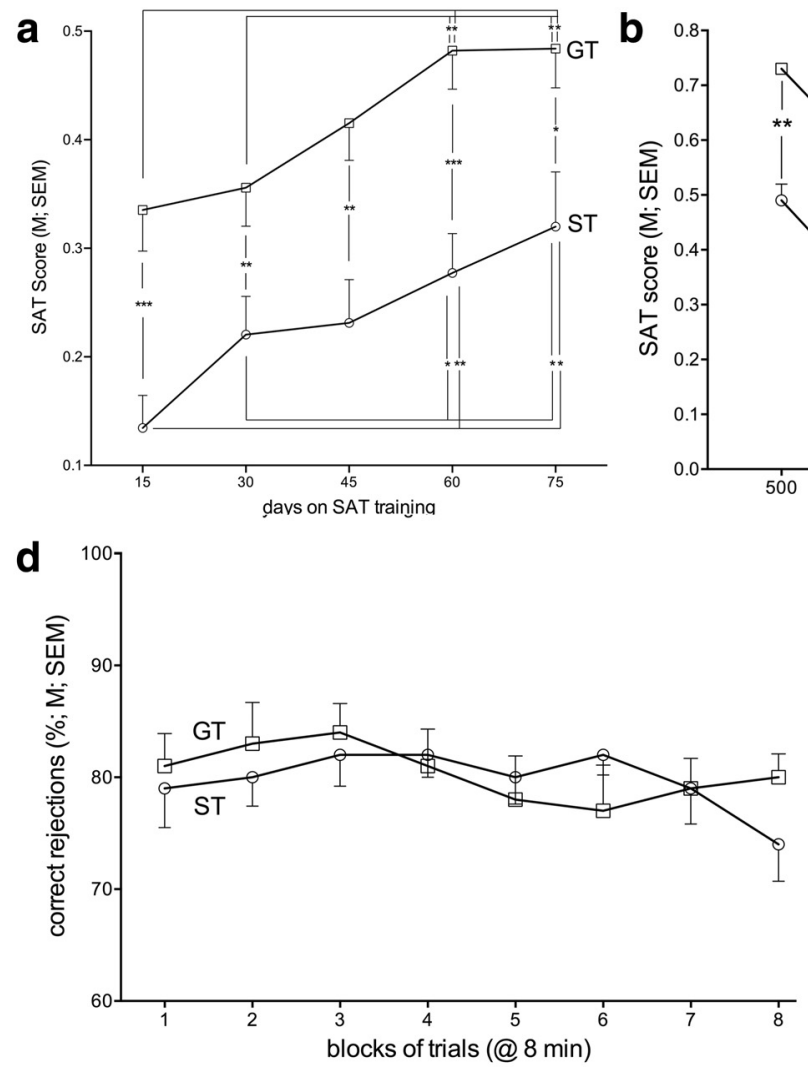
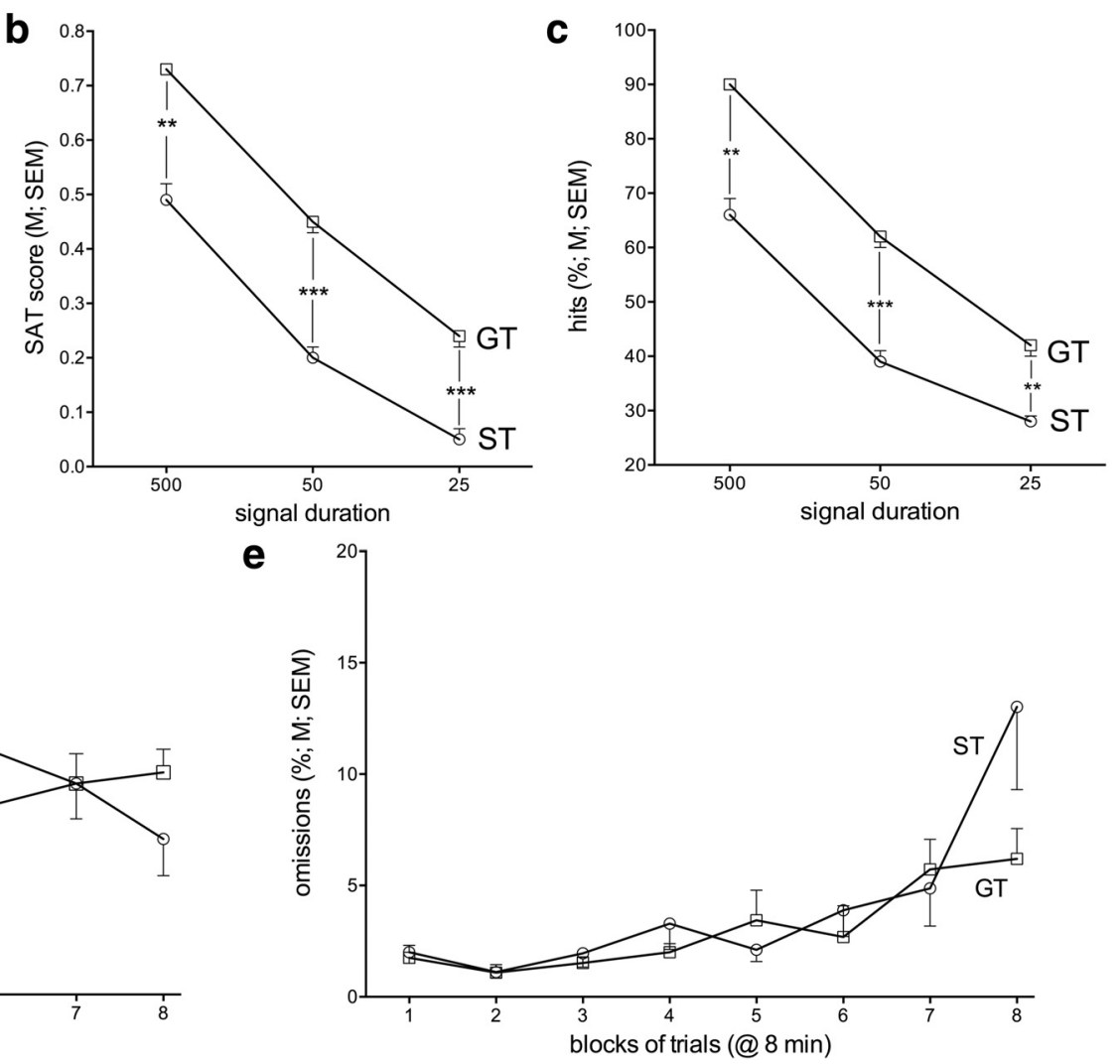

Figure 2. Acquisition of the Sustained Attention Task (SAT) and asymptotic performance. $\boldsymbol{a}$, In terms of overall performance, as indicated by the SAT score, STs ( $n=12$ ) consistently performed at lower levels than GTs $(n=12)$ throughout 75 SAT sessions (note that SAT scores in $\boldsymbol{a}$ are averaged over all signal durations). Both STs and GTs improved their later performance when compared with the initial 30 sessions; performance did not further improve after 45 SAT sessions. $\boldsymbol{b}$, Asymptotic SAT scores as a function of signal duration and group (data from sessions 58 - 60 ). $\boldsymbol{c}$, The overall lower performance by STs, shown in $\boldsymbol{b}$, reflected their lower hit rate. $\boldsymbol{d}$, Nonsignal trial performance did not differ between STs and GTs. All rats generated less correct rejections during the last four blocks of trials when compared with blocks 3 and 4 (see main effect of block and post hoc comparisons in Results). $\boldsymbol{e}$, Errors of omissions did not differ between STs and GTs. All rats omitted $<15 \%$ of the trials and omissions increased for all animals toward later block of trials (see main effect of block in Results). In all graphs, stars indicate the results of multiple-comparisons conducted on the basis of significant main effects and interactions from ANOVAs that are described in Results $\left({ }^{*} p<0.05,{ }^{* *} p<0.01,{ }^{* * *} p<0.001\right)$.

Finally, the PCA index of STs, but not of GTs, correlated with the overall measure of performance (SAT score; STs, Pearson's $r=0.61, p=0.003$; GTs, $r=$ $0.35, p=0.15$ ) (Fig. 3). This correlation indicates that, in STs, a greater propensity for sign tracking was correlated with overall lower attentional performance.

\section{Reliability of SAT performance difference: cohort 2}

As detailed in methods, SAT training for a second cohort of animals began approximately 1 year after obtaining the evidence from cohort 1 described above (see Materials and Methods for detailed description of the number of screened animals and STs/GTs yields per cohort). The animals of cohort 2 required as many sessions as cohort 1 to reach the final task stage (mixed model ANOVA on the effects of cohort and phenotype (between-subject factors): main effects and interaction; all $F<3.44$; all $p>0.07)$. The analysis of asymptotic performance (SAT scores varying $<20 \%$ over 3 consecutive sessions) of the two phenotypes (STs, GTs) and cohorts indicated a main effect of

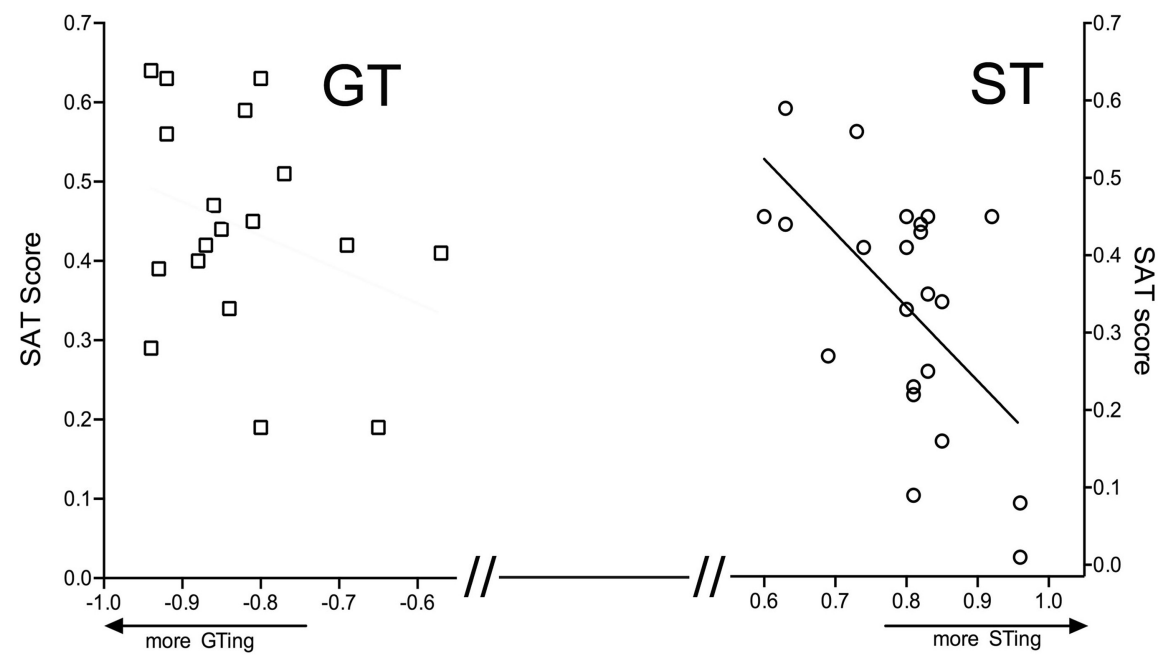

Figure 3. Correlation between SAT performance and PCA scores. This analysis was based on data from all animals that performed at least 45 sessions on the SAT (SAT scores from session 45 were used for this analysis). In STs (circles; $n=22$ ), SAT scores (averaged over signal duration) correlated significantly with the PCA index, that is, the greater the degree of sign tracking the lower their attentional performance (see Results). No correlation was found between the PCA index of GTs (squares; $n=18$ ) and their SAT levels of performance.

phenotype $\left(F_{(1,94)}=5.19, p=0.025 ; \mathrm{M}\right.$, SEM: STs, $0.35 \pm 0.02$, GTs: $0.42 \pm 0.02 ;)$ but no effect of cohort and no interaction between the two factors (both $F<0.47$, both $p>0.50$ ). Moreover, as in cohort 1 , lower SAT scores in STs of cohort 2 were 
again correlated with PCA scores (Pearson's $r=0.33, p=0.04$; GTs: $r=0.16, p=0.19)$. Thus, the lower attentional performance by STs and the correlation between sign tracking and SAT performance were reproduced in a second cohort of rats that was obtained, screened, trained, and tested over one year after the first cohort.

\section{Microanalysis of SAT performance}

SAT performance data (cohort 1) were further inspected and analyzed post hoc to gain additional insight into the nature of the SAT performance difference between STs and GTs and to reveal potential, overt behavioral mechanisms which may have contributed to the relatively lower hit rate in STs.

\section{Performance fluctuation}

Inspection of the animals' performance across trial blocks and sessions suggested that performance levels fluctuated to a greater extent in STs than GTs. To classify levels of performance, SAT scores below chance level $(<0.17)$ were defined as "poor performance" and SAT scores threefold higher than this threshold $(>0.51)$ were classified as "good performance" (intermediate performance: $>0.17<0.51$; see Materials and Methods) (Fig. $4 a$ ). Furthermore, blocking the data from a session ( $60 \mathrm{~min}$ ) into 3 min blocks, each containing $\sim 15$ trials, effectively captured these fluctuations in performance (Fig. $4 b$ shows data from two individuals, an ST and a GT, and illustrates the greater fluctuation of performance in STs).

We first determined the number of times per session STs and GTs shifted to a good, intermediate, or poor performance period (in 3 min units), regardless of the total duration of uninterrupted performance at these levels. STs and GTs entered good or intermediate performance periods at a similar frequency. In contrast, STs entered poor performance periods significantly more frequently than GTs (Fig. 4c) (Mann-Whitney test with $\alpha=0.05 / 3$; good, $\mathrm{U}=60.50, p=0.35$; intermediate, $\mathrm{U}=74.50, p=0.85$; poor, $\mathrm{U}=23.00, p=0.002<0.016$ ).

We also determined the longest, uninterrupted periods during which animals maintained good, intermediate, or poor performance. As illustrated in Figure 4d, STs exhibited longer maximum periods of poor performance, lasting 9 min (median) or longer for more than half of these periods. In contrast, in GTs, poor performance periods ended in most cases after just $3 \mathrm{~min}$. Furthermore, GTs performed at the good level for longer than STs, maintaining uninterrupted good performance for $>9 \mathrm{~min}$, contrasting with 6 min in STs (medians); however, this latter difference was not statistically significant (Fig. $4 d$; Mann-Whitney test with $\alpha=0.05 / 3$; good, $\mathrm{U}=46.50, p=0.08$; intermediate, $\mathrm{U}=73.50, p=0.81$; poor, $\mathrm{U}=33.00, p=0.014<0.016)$.

These analyses indicated that STs more frequently and for longer periods in time shifted to poor performance. We determined next whether these shifts to poor performance periods were mostly gradual, via a period of intermediate performance, or whether STs and GTs differed with regard to the frequency of direct downshifts (directly from good to poor, with no intermediate period). STs shifted directly from good to poor performance almost twice as frequently as GTs [percentage direct downshifts; GTs, 18.31\%; STs, 33.78\%; Fisher's exact test (over absolute direct vs indirect shifts), $p=0.039$ ]. In contrast, the proportion of direct upshifts, relative to upshifts via an intermediate period, did not differ between STs and GTs $(p=0.36)$. Collectively, compared with GTs, STs shifted more frequently and for longer periods from good to poor performance, and these performance downshifts more often were direct, as opposed to involving a period of intermediate performance.

\section{Microbehavioral predictors of shifts to poor performance and errors}

The analyses described above indicated that compared with GTs, SAT performance by STs was characterized by more frequent and direct shifts from good to poor performance and longer periods of poor performance. However, STs were also able to perform at the "good" level, indicating that the two groups did not differ in their essential ability to monitor signal status and execute signal status-dependent response rules. To determine potential, overt behavioral correlates of the performance of STs and GTs we further inspected 3 min periods of good and poor performance. Based on the speculation that STs may produce more misses because they may be positioned in front of the miss/correct rejection lever and are less able to withhold responding and shuttling to the opposite (hit-) lever during signal trials, we categorized the animals' position (in front of the hit/false alarm lever, correct rejection/miss lever, at water port, or away from the intelligence panel) at $1 \mathrm{~s}$ before a signal or, in nonsignal trials, extension of the levers. Furthermore, for this analysis, from each rat we selected 30 trials that constituted a direct shift from good to poor performance (the last 15 at the good level and the first 15 at the poor level).

As illustrated in Figure 5, both STs and GTs were positioned primarily at the water port. The frequency to orient toward the water port (located under the signal source) was significantly higher in STs than in GTs (Mann-Whitney test with $\alpha=0.05 / 4$, $\mathrm{U}=26.50, p=0.0093<0.0125$; for the other three locations: $p>$ 0.0125 ). The positional preferences of STs and GTs did not differ as a function of performance level (Wilcoxon matched-pairs signed-ranks test; all four $p>0.19$ ). Thus, except for the finding that STs are more reliably positioned at the water port, this analysis did not reveal an overt behavioral correlate of the relatively lower level of performance in STs.

\section{SAT performance-associated increase in prefrontal ACh levels}

The results of the analyses of SAT performance suggested that the relatively lower attentional performance in STs reflects a rather "pure" weakness in the cognitive control of attention (Sarter and Paolone, 2011), as opposed to a fundamentally lower ability to comply with the task demands on signal status monitoring, response rule processing and behavioral execution of the response, the need to suppress competitive behaviors, or a lower motivation to perform over a relatively long period in time. We previously demonstrated that SAT performance is associated with increases in cholinergic activity, and that levels of cholinergic activity are correlated with demands on the cognitive control of performance, as opposed to indexing levels of SAT performance (Kozak et al., 2006, 2007; St. Peters et al., 2011b). Therefore, the hypothesis that STs exhibit a lower level of attentional control than in GTs predicted that SAT-associated extracellular ACh levels in prefrontal cortex of STs is lower than levels measured in GTs.

Absolute basal levels of ACh in dialysate did not differ between STs and GTs $\left(t_{(12)}=0.75 p=0.32\right.$; GTs, $5.26 \pm 0.44 \mathrm{fmol} / 12 \mu \mathrm{l}$; STs: $5.50 \pm 0.59 \mathrm{fmol} / 12 \mu \mathrm{l})$. Therefore, task performanceassociated changes in $\mathrm{ACh}$ release were expressed as percentage change from basal levels (Fig. 6a). Mixed-model ANOVA on the effects of phenotype (STs, GTs) and blocks of trials [or collection interval (within-subject factor)] (Fig. 6a,b, T1-T8) on SAT- 
a

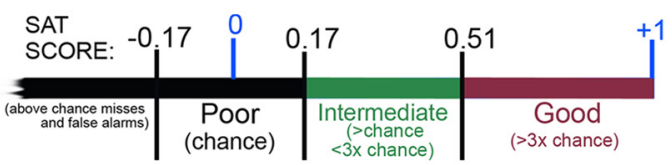

C

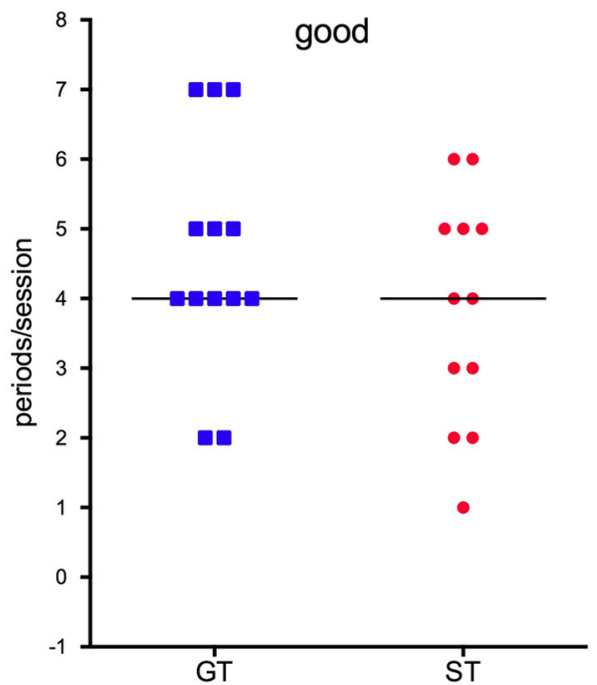

d

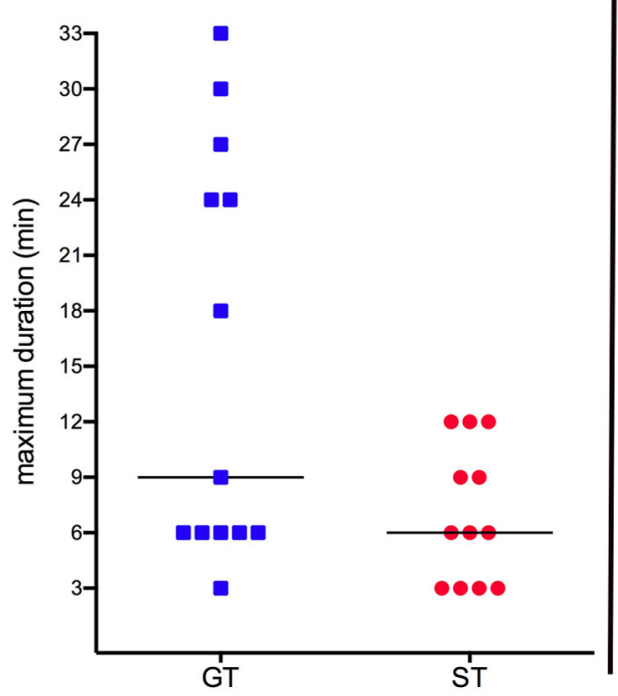

b
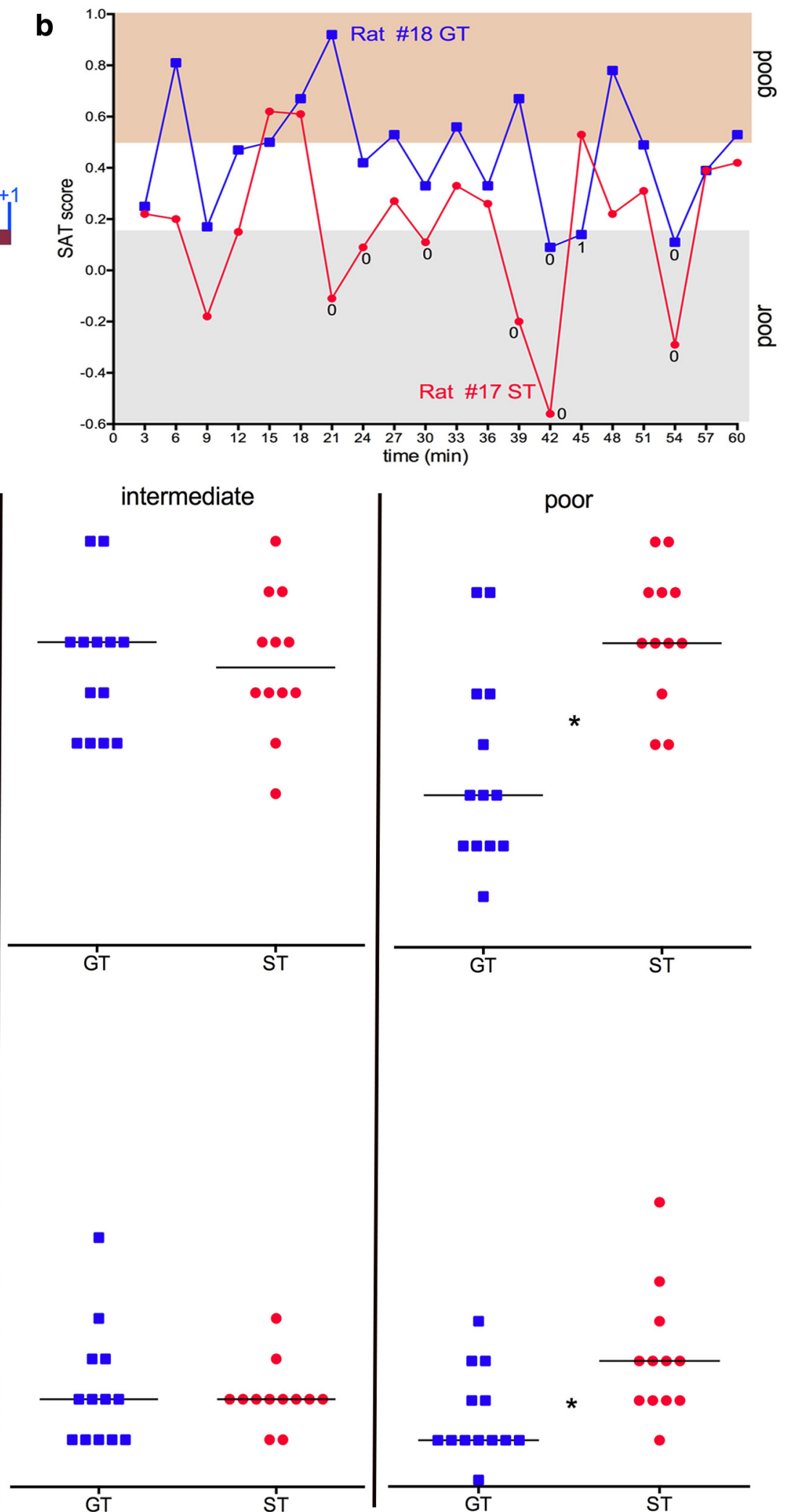

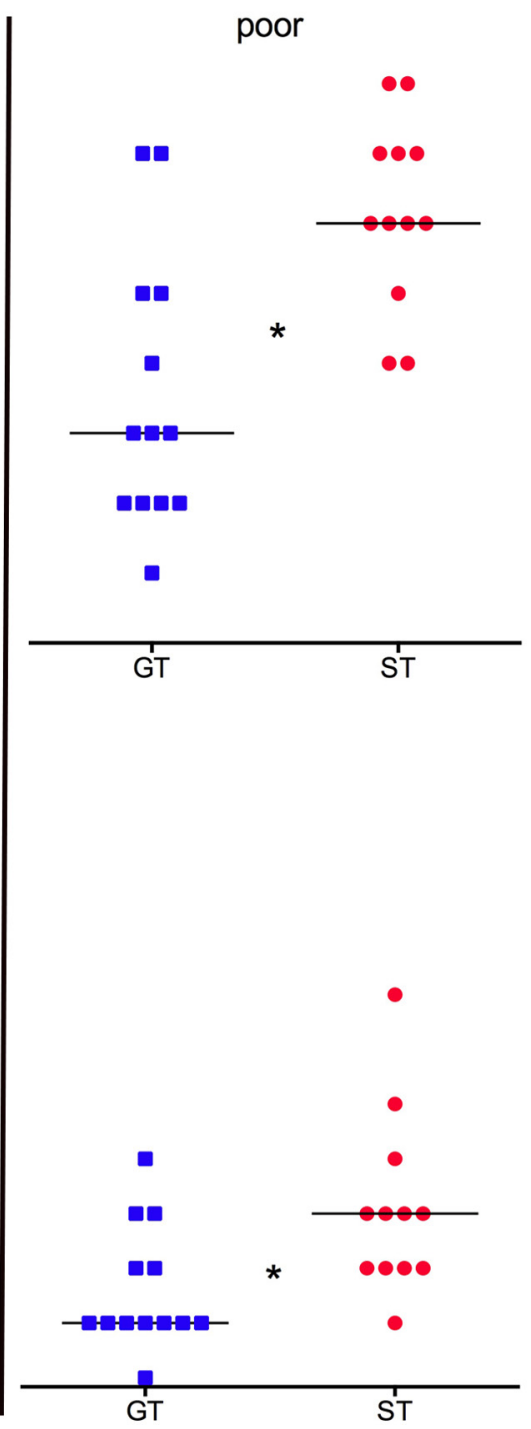

Figure 4. Fluctuation of attentional performance in STs. $\boldsymbol{a}$, Illustration of how categorization of 3 min performance periods were classed as poor, intermediate or good (see Materials and Methods). $\boldsymbol{b}$, Illustration of the greater fluctuation of SAT performance by a representative STs relative to a representative GTs across 3 min blocks of 60 min of SAT session (numbers near data points in the poor performance zone-lower shaded part-indicate the number of omissions for these individual 3 min blocks, confirming that poor performance was not associated with numerous omissions). c, Number of periods of good, intermediate, and poor performance by STs and GTs (medians and scatter). The number of good and intermediate performance periods did not differ between STs and GTs ( $n=6$ per phenotype). However, STs initiated a greater number of periods of poor performance (right). $\boldsymbol{d}$, The maximum duration of poor performance periods (right) was longer in STs compared with GTs. Although the data also suggest that GTs sustained longer periods of good performance (left), this difference remained insignificant (see Results for statistical findings). 


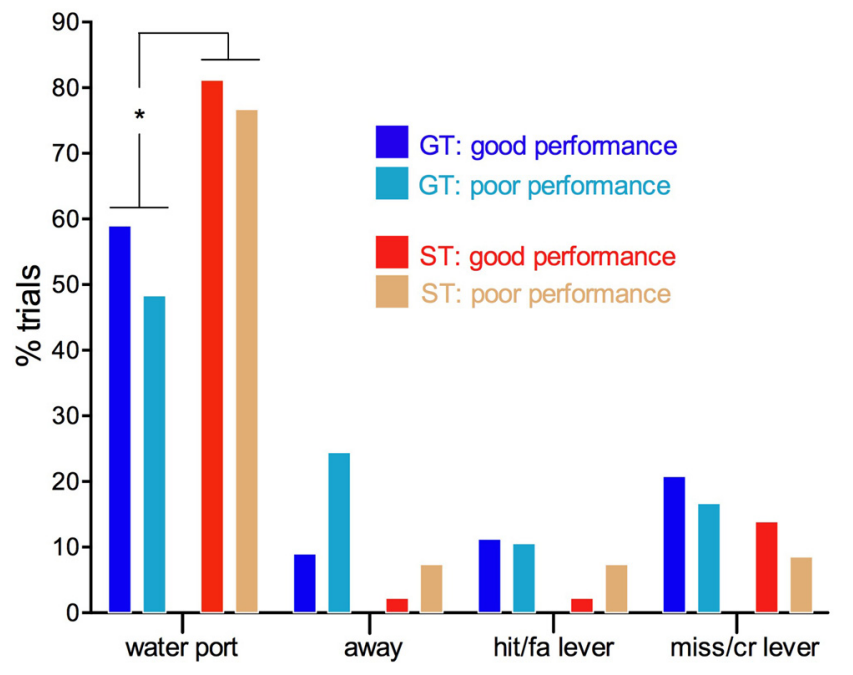

Figure 5. Microbehavioral characteristics of good versus poor SAT performance. The animals' position at $1 \mathrm{~s}$ before a signal and, in nonsignal trials, at $1 \mathrm{~s}$ before lever extension was determined based on video analysis of a total of 30 trials involving a transition from good to poor performance (as defined in Fig. 4a). Specifically, this analysis included the last 15 trials of good performance and the first 15 trials of poor performance that constituted such a transition ( $n=$ 6 per phenotype). Positions were categorized as (1) in front of or at the water port (located below the signal source), (2) oriented "away" from the intelligence panel, (3) in front of and oriented toward the (still retracted) hit /false alarm (fa) lever, or (4) the miss/correct rejection (cr) lever. The graph depicts the results of the analysis of all trials, regardless of response accuracy or trial type (signal or nonsignal). All animals were most frequently positioned in front of or at the water port, with STs positioned at the ports significantly more frequently than GTs (see Results). For the residual number of trials, animals of either group were equally likely to be positioned at any of the other three locations. Likewise, the distribution of positions for GTs and STs did not differ between good and poor performance periods. Thus, there was no overt behavioral correlate of the relatively lower SAT performance by STs or of poor versus good performance in either group.

associated increases in ACh levels indicated a significant main effect of phenotype $\left(F_{(1,12)}=32.23, p<0.0001\right.$; GTs, $204.37 \pm$ $10.87 \%$; STs, $70.68 \pm 6.35 \%$ ) but no effect of collection interval and no interaction between the two factors (both $F<1.04$; both $p>0.40$ ) (Fig. 6a). Notably, previously reported SAT performance-associated ACh levels in groups of unscreened rats correspond with present ACh levels when averaged over STs and GTs (Kozak et al., 2006).

Although SAT performance while tethered and dialyzed typically is lower and more variable compared with regular SAT sessions, SAT scores again were lower in STs (main effect of phenotype, $F_{(1,14)}=5.30, p=0.037$ ) (Fig. 6b). Performing under dialysis conditions impaired STs more severely than GTs (mean SAT scores from regular sessions, taken from above: GTs, 0.42; STs, 0.35; while dialyzed: GTs, 0.40; STs, 0.19). The analysis of SAT performance also indicated an interaction between the effects of task block and phenotype $\left(F_{(7,98)}=3.62, p=0.008\right)$, reflecting that the higher level of performance by GTs reached significance only during blocks 2 and $4\left(t_{(14)}=4.41\right.$ and 2.37, respectively; $p=0.001$ and 0.03 , respectively). It is noteworthy that GTs, but not STs, tended to exhibit further increases in ACh levels toward the end of the session, coinciding with a trend for improvement of performance after declining during earlier blocks 4 and 5, and thus perhaps reflecting the ability of GTs, but not STs, to activate attentional control mechanisms to counteract performance decline (St. Peters et al., 2011b).

The finding that SAT-associated levels of ACh release differed robustly between STs and GTs was reproduced in a small number of rats from cohort 2 . In STs $(n=3)$, SAT-associated increases in
ACh release were $73.39 \pm 8.96 \%$ (M; SEM) whereas levels in GTs $(n=2)$ reached $180.18 \pm 19.48 \%$, consistent with the data from animals from cohort 1 and shown in Figure $6 a$.

\section{Pharmacological enhancement of SAT performance in STs Stimulation of $\alpha 4 \beta 2^{*} n A C h R s$}

Levels of SAT performance-associated ACh release are thought to modulate cortical mechanisms mediating the detection of cues. Furthermore, this modulatory effect of ACh involves stimulation of $\alpha 4 \beta 2^{\star} \mathrm{nAChRs}$ expressed by thalamic inputs to cortex (Parikh et al., 2008, 2010; Hasselmo and Sarter, 2011). As the lower levels of SAT performance-associated ACh release observed in STs insufficiently modulate these cortical mechanisms, we hypothesized that pharmacological stimulation of these receptors improves SAT performance in these rats. The effects of administration of the relatively selective $\alpha 4 \beta 2^{\star} \mathrm{nAChR}$ agonist ABT-089 $(0.02,0.10 \mathrm{mg} / \mathrm{kg})$ confirm this hypothesis. A mixed model ANOVA on the effects of phenotype (STs, GTs; between-subject factor) and dose, block of trials and signal duration (withinsubject factors) on SAT scores reproduced the lower SAT performance by STs (main effect of phenotype; $F_{(1,23)}=11.61$, $p=0.002)$ and indicated a significant interaction between the effects of phenotype and dose $\left(F_{(2,46)}=3.45, p=0.040\right.$; main effect of dose and all other interactions involving dose: all $F<$ 1.67 , all $p>0.061$ ). Pairwise comparisons indicated that the lower dose of ABT-089 significantly enhanced the performance of STs compared with the effects of vehicle and the higher dose of the agonist in STs. Likewise, the lower dose of ABT-089 attenuated the performance difference between STs and GTs (Fig. $7 a$ ). In contrast, administration of ABT-089 did not affect the performance of GTs.

Secondary analyses of the effects of ABT-089 on hits and correct rejections indicated that both measures tended to contribute to the significant ABT-089-induced improvement of SAT performance. First, the analysis on hits indicated a trend for a significant interaction between the effects of dose, group and signal duration $\left(F_{(4,92)}=2.46, p=0.051\right)$, reflecting that the lower, but not the higher, dose of ABT-089 tended to improve the hit rate to longest signals in STs while reducing hits to long and medium-length signals in GTs (see Fig. 7b). Additionally, there was a trend for ABT-089 to improve correct rejections in all animals during the first half of the session (block by dose: $F_{(14,322)}=1.79, p=$ 0.050) (Fig. 7c). Post hoc ANOVAs on the effects of dose per block suggested that ABT-089 benefited the correct rejection rate in block $3\left(F_{(2,22)}=3.87, p=0.036\right)$ and that both doses of ABT-089 were effective during this block (both $t>2.24$, both $p<0.046$ ) (Fig. 7c).

ABT-089 also reduced omissions in all animals (main effect of dose: $\left.F_{(2,46)}=6.70, p=0.005\right)$, further lowering the already low rate of errors of omission observed in vehicle-treated rats (vehicle, $6.15 \pm 0.73 \%$; ABT-089, $0.02 \mathrm{mg} / \mathrm{kg}, 2.92 \pm 0.42 \%$, $t_{(12)}=3.53, p=0.004 ; 0.01 \mathrm{mg} / \mathrm{kg}, 3.11 \pm 0.40 \%, t_{(12)}=2.09$, $p=0.058)$.

The beneficial effect of the lower dose of ABT-089 in STs was also apparent in the analysis of periods of good, intermediate or poor performance (defined above) (Fig. 4a). Administration of ABT-089 reduced the total time STs performed at the poor level (Wilcoxon $\mathrm{W}=114.00, p=0.003<0.05 / 3$; good, $p=0.33$; intermediate, $p=0.85$ ) (Fig. $7 d$ ). We also inspected the shifts from poor to good performance in STs and observed that following administration of the lower dose of ABT-089, STs less frequently shifted directly from poor to good performance (vehicle, $32.46 \%$; ABT, 22.53\%) and more frequently shifted from inter- 
mediate to good performance $(25.97 \%$ vs $40.84 \%$; calculated over events of all 3 categories: $\chi^{2}=8.42, p=0.015$ ).

Nicotine and amphetamine

We also determined the effects of the nonselective $\mathrm{nAChR}$ agonist nicotine (0.02 and $0.4 \mathrm{mg} / \mathrm{kg}$ ) and the psychostimulant amphetamine $(0.25$ and $0.75 \mathrm{mg} / \mathrm{kg})$ on SAT performance in STs and GTs. Mixed model ANOVAs on the effects of the two drugs (analyzed separately), with the factors phenotype (between-subject factor) and dose, block of trials and, where applicable, signal duration (within-subject factors), confirmed the performance difference between STs and GTs (main effect of phenotype, both $F_{(1,20)}>5.16$, both $p<0.034$; data not shown). However, similar to our prior reports on the effects of nicotine in unscreened rats (Howe et al., 2010), nicotine did not affect performance (main effect of drug and interactions involving drug, all $\mathrm{F}<1.89, p>0.11$ ). The analysis of the effects of amphetamine indicated a main effect of drug $\left(F_{(2,40)}=10.95, p<\right.$ $0.001)$ but no interaction with group $\left(F_{(2,40)}=0.97, p=0.39\right)$. Post hoc multiplecomparisons indicated that this main effect of amphetamine was due to an impairment in performance after the administration of the higher dose (SAT scores: vehicle, $0.41 \pm$ $0.02 ; 0.25 \mathrm{mg} / \mathrm{kg}, 0.48 \pm 0.01, t_{(10)}=1.68$, $p=0.12 ; 0.75 \mathrm{mg} / \mathrm{kg}, 0.32 \pm 0.02 ; t_{(10)}=$ 2.28, $p=0.046$ ).

\section{SAT-associated $\mathrm{ACh}$ release after treatment with ABT-089}

Administration of the lower dose of ABT089 improved SAT performance in STs (above). This finding is consistent with the hypothesis that stimulation of $\alpha 4 \beta 2^{*}$ nAChRs mimics and amplifies the modulation of cortical attention circuitry and enhances control of attention. We were therefore interested in monitoring performance-associated, endogenous ACh release in the presence of ABT-089, speculating that levels of ACh release may be altered as a result of feedback between the effects of the drug at $\alpha 4 \beta 2^{\star} \mathrm{nAChRs}$ and the recruitment of cholinergic neurons via local cortical as well as distributed circuitry, including corticomesolimbic-basal forebrain connec-

tions (St. Peters et al., 2011b). We therefore measured extracellular ACh levels in the presence of the lower dose of ABT-089 (0.02 mg/ $\mathrm{kg}$ ) in SAT-performing STs and GTs.

Figure $8 a$ depicts ACh levels in STs and GTs in the presence and absence of ABT-089 (0.02 mg/kg). Mixed-model ANOVA on the effects of phenotype and collection interval (T1-T8; withinsubject factor) on ACh levels during SAT indicated a main effect of phenotype $\left(F_{(1,8)}\right)=13.21, p=0.007$; GTs: $181.36 \pm 8.83 \%$; STs: $81.53 \pm 7.30 \%)$, confirming that STs released less ACh dur-
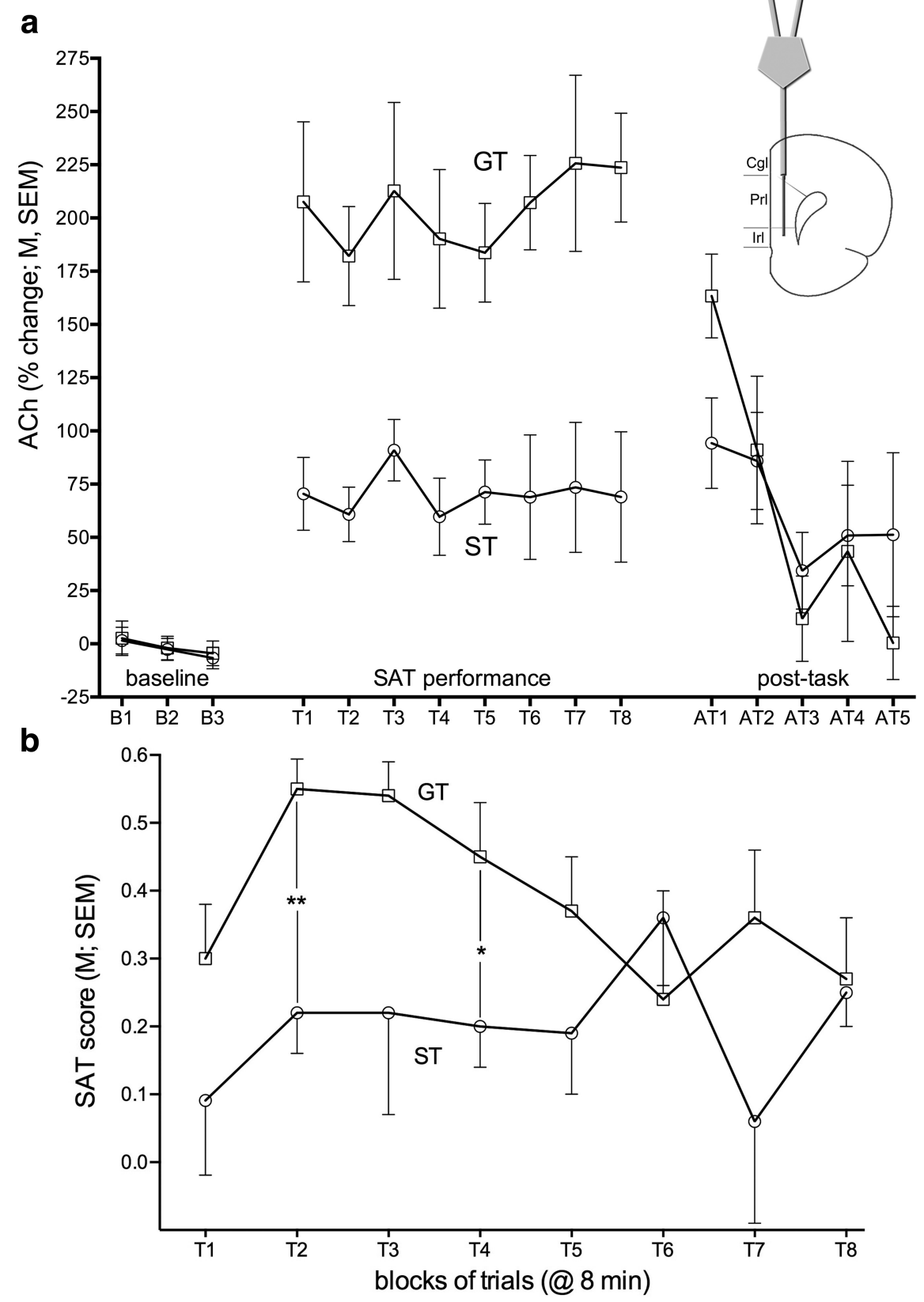

Figure 6. SAT-performance-associated increases in extracellular ACh levels in the medial prefrontal cortex of GTs and STs. The inset depicts the approximate position of the active dialysis membrane in the prelimbic cortex. Cgl, Cingulate cortex; Prl, prelimbic cortex; Irl, infralimbic cortex. Note that the majority of the active membrane of the probe was located in the prelimbic region. $\boldsymbol{a}$, Absolute basal ACh release levels did not differ between the groups (see Results) and thus data were expressed as percentage change from baseline. ANOVA indicated a main effect of group but no interaction with block of trials (or collection intervals). SAT performance-associated increase in ACh release was significantly higher in GTs $(n=7)$ compared with STs $(n=7)$. The SAT scores in $\boldsymbol{b}$ were plotted over blocks of trials (abscissa) to correspond with microdialysis collections (T1-T8) in $\boldsymbol{a}$ (note that statistical analysis indicated a main effect of phenotype but no interaction between this factor and block of trials; see Results).

ing SAT performance than GTs. Administration of ABT-089 differently affected performance-associated ACh release as indicated by a significant interaction between the effects of drug and phenotype $\left(F_{(1,8)}=5.47, p=0.048\right.$; no interaction between drug, group and block; $F_{(7,56)}=0.97, p=0.45$ ) (Figure $8 a$ ). Because ABT-089 lowered ACh levels in GTs and increased ACh levels in STs, the group difference was abolished following treatment (percentage change ACh: GTs + vehicle, 199.22 $\pm 23.88 \%$; STs + vehicle, $72.27 \pm 12.91 \% ; t_{(12)}=4.68, p=0.001$; GTs $+\mathrm{ABT}$, $156.8 \pm 27.44 \%$; STs + ABT, $101.49 \pm 21.98 \%)$. 

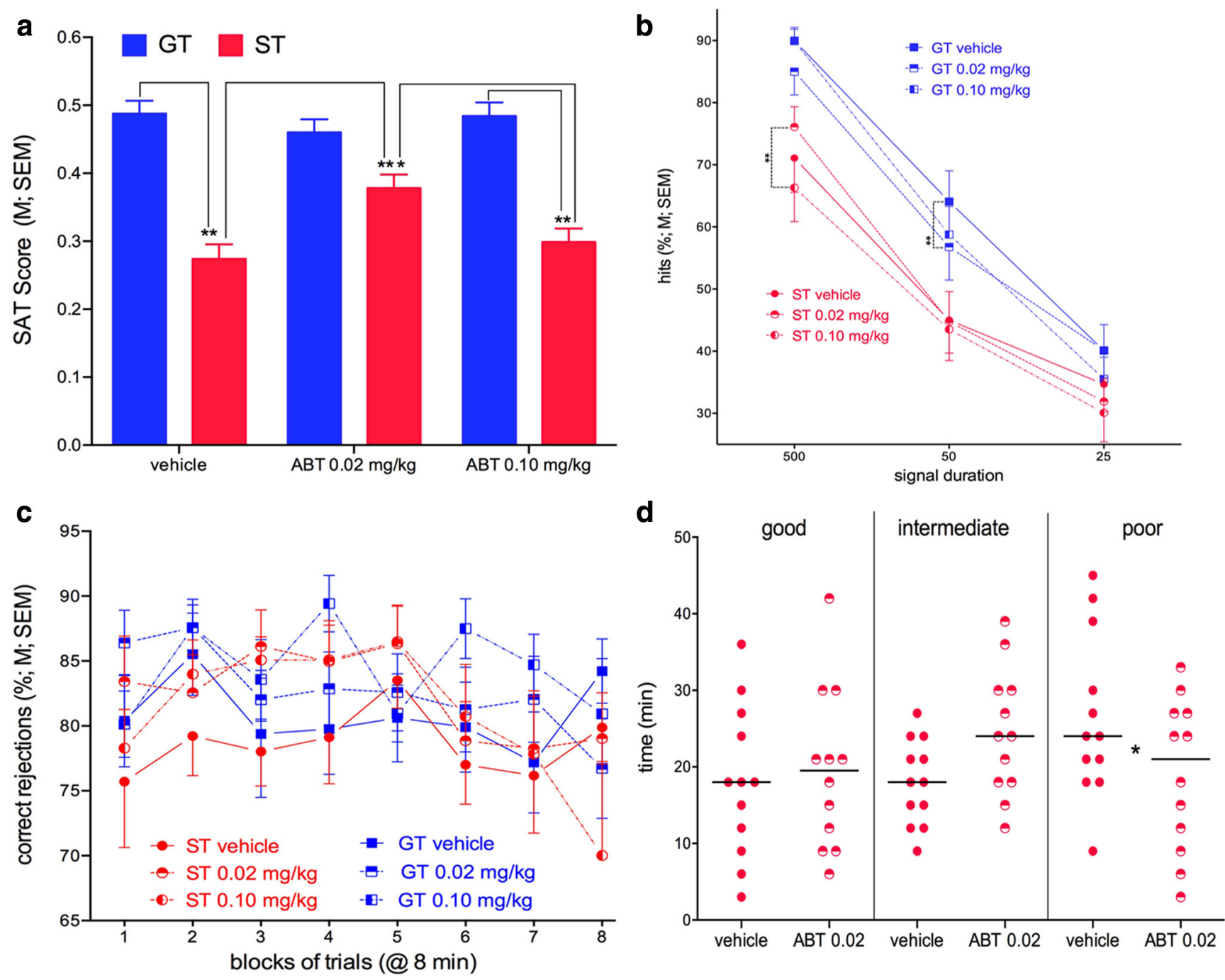

Figure 7. Enhancement of STs' SAT performance by ABT-089. $\boldsymbol{a}$, The SAT scores of GTs $(n=13)$ and STs $(n=12)$ after the administration of vehicle or ABT-089. ANOVA indicated a significant interaction between the effects of dose and group on SAT scores (see Results). Pairwise comparisons revealed that the lower dose of ABT-089 enhanced SAT performance in STs compared with vehicle or the higher dose; likewise, this lower dose abolished the difference between STs and GTs seen after the administration of vehicle. $\boldsymbol{b}, \boldsymbol{c}$, The significant effect of the lower dose of ABT-089 on SAT scores in STs reflected trends for increasing their hit rates to longest signals ( $\boldsymbol{b}$ ) and an increase in correct rejections for all rats (c) (indications of significant post hoc comparisons in $\boldsymbol{b}$ are exploratory as they were based on trends as revealed by ANOVA; see Results). $\boldsymbol{d}$, In STs, administration of ABT-089 (lower dose) significantly decreased their total time spent performing poorly (as defined in Fig. 4a) (each data point represents the total time on that level for one rat). The ABT-089-induced increases in the time spent at the intermediate and good level did not reach significance.

As mentioned, the experimental manipulations associated with conducting microdialysis, particularly the tethering of rats, typically lowers levels and increases the variability of SAT performance. Despite this issue, this experiment again confirmed the lower level of performance of STs compared with GTs (main effect of phenotype: $F_{(1,8)}=17.62, p=0.003$ ). Consistent with the earlier observation that STs were more severely affected by the dialysis conditions (above), ABT-089 failed to improve performance of STs while undergoing microdialysis (main effect of dose and all interactions involving dose: all $F<4.30$, all $p>0.07$ ) (Fig. 8b).

\section{Discussion}

These experiments generated the following main results. (1) STs performed poorly on the SAT relative to GTs, as indicated by a lower number of hits in STs than GTs. Furthermore, in STs, strong sign tracking behavior was negatively correlated with SAT performance. These findings were replicated in a second cohort of animals tested 1 year after the first, suggesting they are reliable.
(2) During SAT performance extracellular medial prefrontal ACh levels increased less in STs than GTs. (3) Administration of the $\alpha 4 \beta 2^{\star}$ nAChR partial agonist ABT-089, but not of nicotine or amphetamine, improved SAT performance in STs. In the presence of ABT-089, SAT-associated increases in prefrontal ACh release were attenuated and augmented in GTs and STs, respectively, suggesting, as will be discussed further below, feedback mechanisms designed to stabilize the attentional control mechanisms that are mediated via the cortical cholinergic input system.

\section{Behavior}

We asked whether variation in the propensity to attribute incentive salience to reward cues (STs) is related to variation in attentional control in rats. We found that, relative to GTs, STs performed poorly on the SAT, scoring a lower number of hits (reporting misses to more signals). Importantly, the relatively poor performance of STs was due to greater and more frequent shifts between periods of good performance directly to periods of poor performance. This latter point is significant because it shows 
that STs can perform as well as GTs, indicating that STs could adequately monitor signal status and execute correct responses. Furthermore, omissions were low in both groups, indicating that STs were not less motivated to perform than GTs. STs have been reported to show a greater propensity for impulsive actions (Lovic et al., 2011), but we found no evidence that this was the basis for poor performance here, based on an analysis of the animals' position before hits or misses.

In the absence of overt behavioral correlates and given the greater fluctuation of performance by STs, including their propensity for direct shifts from good to poor performance (without going through a period of intermediate performance), we suggest that the most parsimonious explanation of variation in SAT performance is that it reflects relatively poor cognitive or "top-down" control of attention in STs, including a relatively lower capacity for maintaining task rules and behavioral goals in working memory (or "on-line"), yielding fluctuating performance (for a detailed discussion of this construct, see Sarter and Paolone, 2011). Furthermore, STs may exhibit poorer levels of performance monitoring, specifically error monitoring, consistent with longer periods of poor performance, and the associated tolerance for extended periods of reward loss. In GTs, periods of poor performance remained shorter, suggesting that reward loss may have more reliably activated cognitive control mechanisms, such as enhanced cue processing and distractor filtering, which serve to combat performance decline and foster recovery from poor performance (Sarter et al., 2006). The trend for augmented ACh levels in GTs late in the session and following a performance drop (Fig. 6) is consistent with this notion.

\section{Neurochemistry}

Previous studies have established that good performance on the SAT requires an increase in $\mathrm{ACh}$ in prefrontal cortex during task performance (St. Peters et al., 2011b). In STs, SAT performance was associated with significantly attenuated increases in medial prefrontal ACh release, relative to GTs. We previously demonstrated that SAT performance-associated levels of ACh release are highest when demands on attentional control are high, such as during the presentation of a distractor (St. Peters et al., 2011b). Moreover, higher levels of release were correlated with a less severe distractor impact on performance, supporting the hypothesis that higher levels of cholinergic activity indicate greater attentional control or attentional "effort" (Sarter et al., 2006). Conversely, this hypothesis suggests re-
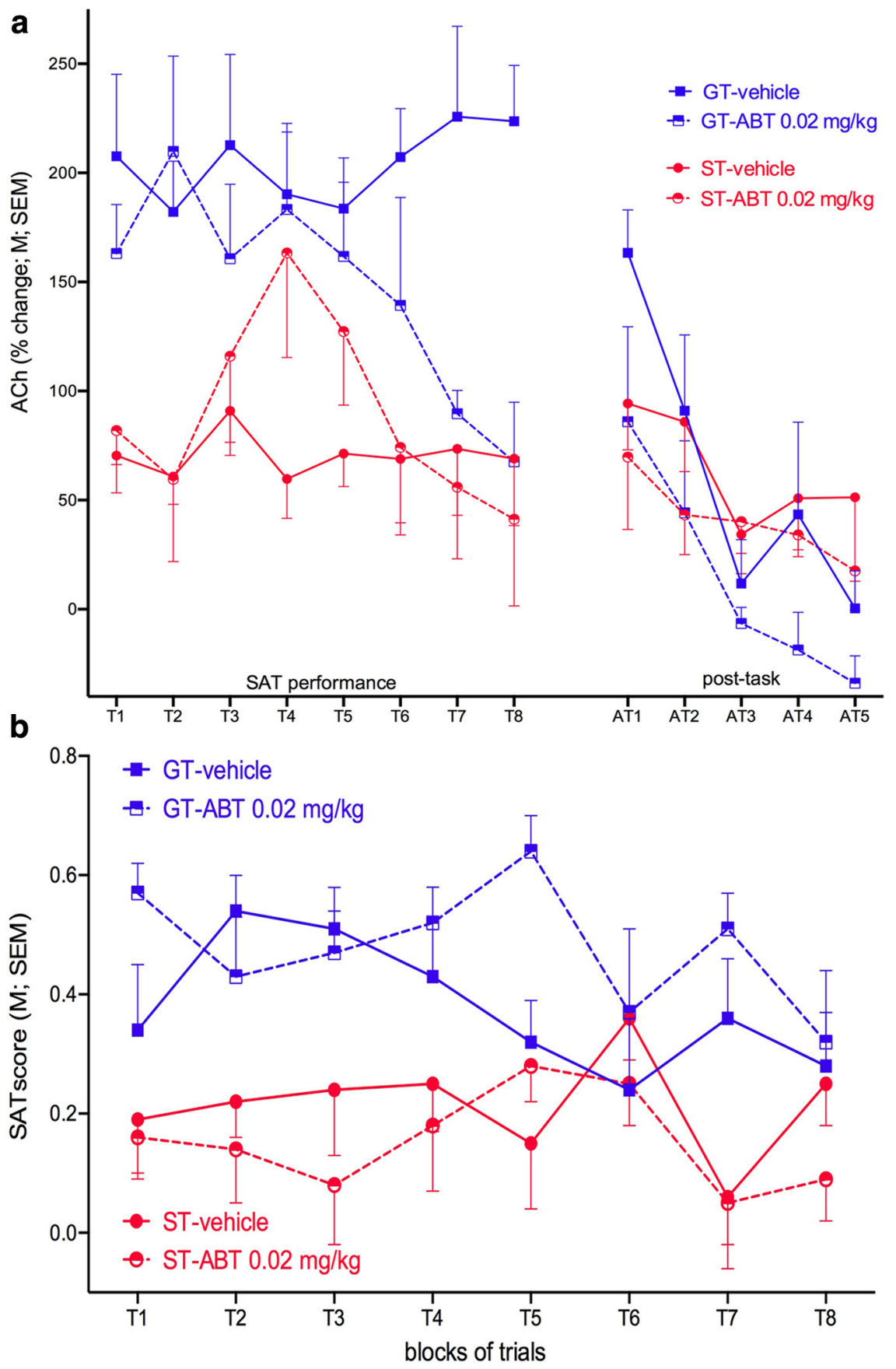

Figure 8. SAT performance-associated ACh release in the presence of ABT-089. $\boldsymbol{a}$, ACh release in STs during SAT sessions with and without treatment of ABT-089 $(0.02 \mathrm{mg} / \mathrm{kg})$. ABT-089 lowered SAT-associated ACh levels in GTs $(n=5)$ toward the end of the session while increasing levels in STs $(n=5)$ during middle blocks of trials. Statistical analysis confirmed that STs released less ACh during SAT than GTs (main effect of group) and a significant interaction between the effects of group and drug, reflecting the opposed effects of ABT-089 in the two groups of animals. $\boldsymbol{b}$, STs again performed at lower levels than GTs; however, because of the detrimental effects of performing under microdialysis conditions, this experiment did not reveal the beneficial performance effects of the drug seen in STs performing the SAT without being tethered (Fig. 7).

duced attentional control in STs compared with GTs. Indeed, in STs the low levels of performance-associated ACh were approaching levels observed in an animal model of schizophrenia that likewise exhibits poor attentional control (Kozak et al., 2007, their Fig. 4).

SAT performance-associated ACh levels are relatively stable across collection intervals, supporting the view that in the absence of performance challenges, levels of attentional control are 
relatively stable (Paolone et al., 2010). Following performance challenges and/or to counteract decreases in performance, higher levels of tonic $\mathrm{ACh}$ are generated, likely due in part to activation of mesolimbic-cholinergic interactions (see below) (St. Peters et al., 2011b). These augmented levels of cholinergic activity are thought to amplify cue-evoked glutamate release from thalamic inputs to the cortex, thereby enhancing cortical mechanisms mediating the detection of signals (for review, see Hasselmo and Sarter, 2011). Thalamic inputs express $\alpha 4 \beta 2^{\star}$ nAChRs and removal of these receptors attenuate nicotine-evoked glutamate release (Parikh et al., 2008, 2010). As $\alpha 4 \beta 2^{*}$ nAChRs do not downregulate as a result of continuous stimulation (e.g., Nashmi et al., 2007; Xiao et al., 2009) these receptors are a promising target for agonist treatment, as confirmed by the efficacy of $\alpha 4 \beta 2^{*}$ nAChR agonists, including ABT-089, in enhancing attentional control in animals and humans (Howe et al., 2010; Apostol et al., 2011).

\section{Pharmacology}

It is especially interesting, therefore, that administration of the $\alpha 4 \beta 2^{*}$ nAChR partial agonist ABT-089, but not of nicotine or amphetamine, improved SAT performance in STs. ABT-089 reduced the time spent performing poorly and increased the frequency with which STs shifted from poor to good levels of performance via a period of performance at an intermediate level, as opposed to direct upshifts. This suggests that this compound acted by enhancing attentional control mechanisms and not, for example, by enhancing basic perceptual capacities. Although the effects of ABT-089 were significant, they were modest, as indicated by (strong) trends only for effects on individual performance measures. Perhaps more efficacious full agonists at these receptors would generate more robust effects (Bain et al., 2013).

In the presence of ABT-089, SAT-associated ACh levels decreased in GTs but increased in STs, yielding a significant interaction between the effects of group and drug on release. In GTs, direct stimulation of nAChR may have led to negative feedback, resulting in a lowering of the normally high levels of SATassociated ACh release toward the end of the session (Fig. 8a). Circuitry mediating such feedback likely involves prefrontalmesolimbic-basal forebrain circuitry (Záborszky and Cullinan, 1992), corresponding with circuits responsible for enhancing cholinergic activity as a result of demands on performance (St. Peters et al., 2011b) and contributing to drug-seeking behavior (Miller and Marshall, 2004; George and Koob, 2010; Bossert et al., 2012; St. Onge et al., 2012; Calu et al., 2013). In STs, the trend for a transient augmentation of SAT-associated ACh release in the presence of ABT-089 may be speculated to reflect a brief recruitment of endogenous control mechanisms, assisted by the drug effect. Unfortunately, as addressed in Results, the experimental conditions associated with dialysis hamper task performance and prevented the demonstration of a performance correlate. The effects of ABT-089 on SAT-associated ACh release illustrate the importance of conducting analyses of neurochemical effects of drugs in task-performing rats, as opposed to measures obtained from passive animals not recruiting the circuitry of interest (Sarter et al., 2007).

\section{Relevance for addiction}

Individuals are most likely to succumb to the temptations afforded by reward cues if they both attribute high motivational value to such cues, and also have difficulty suppressing attention to such cues, and toward less attractive but also less maladaptive cues. We report here that some outbred rats (STs) not only attri- bute considerable incentive salience to reward cues, but also combine this with relatively poor attentional control, due in part to attenuated ability to increase prefrontal ACh during periods of high attentional demand. It is not clear whether poor attentional control and high incentive salience attribution are cognitive and behavioral manifestations of the same trait or of multiple, associated psychological traits, but this combination may render such individuals especially vulnerable to disorders characterized by poor restraint, such as obesity and addiction (Jentsch and Taylor, 1999; Robinson and Berridge, 1993). In addicts, such interactions may be specifically revealed in situations where drug cues foster relapse and where effective attentional control is required to suppress attention to a powerfully attractive drug cue, and to shift behavior to be guided by other less attractive cues.

In STs (but not GTs) a food cue evokes a phasic dopamine signal in the core of the accumbens, and dopamine is necessary for the learning and performance of sign-tracking but not goaltracking behavior (Flagel et al., 2011; Saunders and Robinson, 2012). However, the role of mesolimbic phasic dopamine signaling during attentional performance is unknown. Nevertheless, stimulation of the shell of the nucleus accumbens, but not the core, benefits the cholinergic control of attention (St. Peters et al., 2011b). This raises the possibility that mesolimbic-telencephalic, and dopaminergic-cholinergic interactions differ fundamentally between STs and GTs. Finally, the ability of the $\alpha 4 \beta 2^{*} \mathrm{nAChR}$ partial agonist ABT-089 to at least partially facilitate the performance of STs suggests that pharmacological enhancement of attentional control mechanisms may provide a way to increase the likelihood that an individual foregoes using high incentive, but sometimes costly drug or food cues, in favor of more adaptive alternatives.

\section{References}

Apostol G, Abi-Saab W, Kratochvil CJ, Adler LA, Robieson WZ, Gault LM, Pritchett YL, Feifel D, Collins MA, Saltarelli MD (2011) Efficacy and safety of the novel $\alpha(4) \beta(2)$ neuronal nicotinic receptor partial agonist ABT-089 in adults with attention-deficit/hyperactivity disorder: a randomized, double-blind, placebo-controlled crossover study. Psychopharmacology (Berl) 219:715-725. CrossRef Medline

Apparsundaram S, Martinez V, Parikh V, Kozak R, Sarter M (2005) Increased capacity and density of choline transporters situated in synaptic membranes of the right medial prefrontal cortex of attentional taskperforming rats. J Neurosci 25:3851-3856. CrossRef Medline

Bain EE, Robieson W, Pritchett Y, Garimella T, Abi-Saab W, Apostol G, McGough JJ, Saltarelli MD (2013) A randomized, double-blind, placebo-controlled phase 2 study of $\alpha 4 \beta 2$ agonist ABT-894 in adults with ADHD. Neuropsychopharmacology 38:405-413. CrossRef Medline

Bossert JM, Stern AL, Theberge FR, Marchant NJ, Wang HL, Morales M, Shaham Y (2012) Role of projections from ventral medial prefrontal cortex to nucleus accumbens shell in context-induced reinstatement of heroin seeking. J Neurosci 32:4982-4991. CrossRef Medline

Broos N, Diergaarde L, Schoffelmeer AN, Pattij T, De Vries TJ (2012) Trait impulsive choice predicts resistance to extinction and propensity to relapse to cocaine seeking: a bidirectional investigation. Neuropsychopharmacology 37:1377-1386. CrossRef Medline

Calu DJ, Kawa AB, Marchant NJ, Navarre BM, Henderson MJ, Chen B, Yau HJ, Bossert JM, Schoenbaum G, Deisseroth K, Harvey BK, Hope BT, Shaham Y (2013) Optogenetic inhibition of dorsal medial prefrontal cortex attenuates stress-induced reinstatement of palatable food seeking in female rats. J Neurosci 33:214-226. CrossRef Medline

Decker MW, Bannon AW, Curzon P, Gunther KL, Brioni JD, Holladay MW, Lin $\mathrm{NH}$, Li Y, Daanen JF, Buccafusco JJ, Prendergast MA, Jackson WJ, Arneric SP (1997) ABT-089 [2-methyl-3-(2-(S)-pyrrolidinylmethoxy)pyridine dihydrochloride]: II. A novel cholinergic channel modulator with effects on cognitive performance in rats and monkeys. J Pharmacol Exp Ther 283:247-258. Medline

Demeter E, Sarter M, Lustig C (2008) Rats and humans paying attention: 
cross-species task development for translational research. Neuropsychology 22:787-799. CrossRef Medline

Demeter E, Hernandez-Garcia L, Sarter M, Lustig C (2011) Challenges to attention: a continuous arterial spin labeling (ASL) study of the effects of distraction on sustained attention. Neuroimage 54:1518-1529. CrossRef Medline

Demeter E, Guthrie SK, Taylor S, Sarter M, Lustig C (2013) Increased distractor vulnerability but preserved vigilance in patients with schizophrenia: evidence from a translational sustained attention task. Schizophr Res 144:136-141. CrossRef Medline

Ersche KD, Bullmore ET, Craig KJ, Shabbir SS, Abbott S, Müller U, Ooi C, Suckling J, Barnes A, Sahakian BJ, Merlo-Pich EV, Robbins TW (2010) Influence of compulsivity of drug abuse on dopaminergic modulation of attentional bias in stimulant dependence. Arch Gen Psychiatry 67:632-644. CrossRef Medline

Ersche KD, Barnes A, Jones PS, Morein-Zamir S, Robbins TW, Bullmore ET (2011) Abnormal structure of frontostriatal brain systems is associated with aspects of impulsivity and compulsivity in cocaine dependence. Brain 134:2013-2024. CrossRef Medline

Ersche KD, Jones PS, Williams GB, Turton AJ, Robbins TW, Bullmore ET (2012) Abnormal brain structure implicated in stimulant drug addiction. Science 335:601-604. CrossRef Medline

Field M, Cox WM (2008) Attentional bias in addictive behaviors: a review of its development, causes, and consequences. Drug Alcohol Depend 97:120. CrossRef Medline

Flagel SB, Akil H, Robinson TE (2009) Individual differences in the attribution of incentive salience to reward-related cues: implications for addiction. Neuropharmacology 56:139-148. CrossRef Medline

Flagel SB, Clark JJ, Robinson TE, Mayo L, Czuj A, Willuhn I, Akers CA, Clinton SM, Phillips PE, Akil H (2011) A selective role for dopamine in stimulus-reward learning. Nature 469:53-57. CrossRef Medline

Frey PW, Colliver JA (1973) Sensitivity and responsivity measures for discrimination learning. Learning and Motivation 4:327-342. CrossRef

George O, Koob GF (2010) Individual differences in prefrontal cortex function and the transition from drug use to drug dependence. Neurosci Biobehav Rev 35:232-247. CrossRef Medline

Goldstein RZ, Volkow ND (2011) Dysfunction of the prefrontal cortex in addiction: neuroimaging findings and clinical implications. Nat Rev Neurosci 12:652-669. CrossRef Medline

Greenwald AG, Gonzalez R, Harris RJ, Guthrie D (1996) Effect sizes and p values: what should be reported and what should be replicated? Psychophysiology 33:175-183. CrossRef Medline

Hahn B, Shoaib M, Stolerman IP (2003) Involvement of the prefrontal cortex but not the dorsal hippocampus in the attention-enhancing effects of nicotine in rats. Psychopharmacology (Berl) 168:271-279. CrossRef Medline

Hahn B, Ross TJ, Yang Y, Kim I, Huestis MA, Stein EA (2007) Nicotine enhances visuospatial attention by deactivating areas of the resting brain default network. J Neurosci 27:3477-3489. CrossRef Medline

Hasselmo ME, Sarter M (2011) Modes and models of forebrain cholinergic neuromodulation of cognition. Neuropsychopharmacology 36:52-73. CrossRef Medline

Howe WM, Ji J, Parikh V, Williams S, Mocaër E, Trocmé-Thibierge C, Sarter M (2010) Enhancement of attentional performance by selective stimulation of alpha4beta $2^{\star}$ nAChRs: underlying cholinergic mechanisms. Neuropsychopharmacology 35:1391-1401. CrossRef Medline

Jentsch JD, Taylor JR (1999) Impulsivity resulting from frontostriatal dysfunction in drug abuse: implications for the control of behavior by reward-related stimuli. Psychopharmacology 146:373-390. CrossRef Medline

Kozak R, Bruno JP, Sarter M (2006) Augmented prefrontal acetylcholine release during challenged attentional performance. Cereb Cortex 16: 9-17. CrossRef Medline

Kozak R, Martinez V, Young D, Brown H, Bruno JP, Sarter M (2007) Toward a neuro-cognitive animal model of the cognitive symptoms of schizophrenia: disruption of cortical cholinergic neurotransmission following repeated amphetamine exposure in attentional task-performing, but not non-performing, rats. Neuropsychopharmacology 32:20742086. CrossRef Medline

Lovic V, Saunders BT, Yager LM, Robinson TE (2011) Rats prone to attribute incentive salience to reward cues are also prone to impulsive action. Behav Brain Res 223:255-261. CrossRef Medline
Lucantonio F, Stalnaker TA, Shaham Y, Niv Y, Schoenbaum G (2012) The impact of orbitofrontal dysfunction on cocaine addiction. Nat Neurosci 15:358-366. CrossRef Medline

McGaughy J, Sarter M (1995) Behavioral vigilance in rats: task validation and effects of age, amphetamine, and benzodiazepine receptor ligands. Psychopharmacology (Berl) 117:340-357. CrossRef Medline

McGaughy J, Sarter M (1999) Effects of ovariectomy, 192 IgG-saporininduced cortical cholinergic deafferentation, and administration of estradiol on sustained attention performance in rats. Behav Neurosci 113: 1216-1232. CrossRef Medline

Meyer PJ, Lovic V, Saunders BT, Yager LM, Flagel SB, Morrow JD, Robinson TE (2012) Quantifying individual variation in the propensity to attribute incentive salience to reward cues. PLoS One 7:e38987. CrossRef Medline

Miller CA, Marshall JF (2004) Altered prelimbic cortex output during cueelicited drug seeking. J Neurosci 24:6889-6897. CrossRef Medline

Nashmi R, Xiao C, Deshpande P, McKinney S, Grady SR, Whiteaker P, Huang Q, McClure-Begley T, Lindstrom JM, Labarca C, Collins AC, Marks MJ, Lester HA (2007) Chronic nicotine cell specifically upregulates functional alpha ${ }^{*}$ nicotinic receptors: basis for both tolerance in midbrain and enhanced long-term potentiation in perforant path. J Neurosci 27: 8202-8218. CrossRef Medline

Newhouse PA, Potter AS, Dumas JA, Thiel CM (2011) Functional brain imaging of nicotinic effects on higher cognitive processes. Biochem Pharmacol 82:943-951. CrossRef Medline

Paolone G, Howe WM, Gopalakrishnan M, Decker MW, Sarter M (2010) Regulation and function of the tonic component of cortical acetylcholine release. In: Monitoring molecules in neuroscience (Westerink B, Clinckers R, Smolders S, Sarre S, Michotte Y, eds), pp 363-365. Brussels: Vrije Universiteit Brussels.

Parikh V, Kozak R, Martinez V, Sarter M (2007) Prefrontal acetylcholine release controls cue detection on multiple timescales. Neuron 56:141154. CrossRef Medline

Parikh V, Man K, Decker MW, Sarter M (2008) Glutamatergic contributions to nicotinic acetylcholine receptor agonist-evoked cholinergic transients in the prefrontal cortex. J Neurosci 28:3769-3780. CrossRef Medline

Parikh V, Ji J, Decker MW, Sarter M (2010) Prefrontal beta2 subunitcontaining and alpha7 nicotinic acetylcholine receptors differentially control glutamatergic and cholinergic signaling. J Neurosci 30:35183530. CrossRef Medline

Parikh V, St Peters M, Blakely RD, Sarter M (2013) The presynaptic choline transporter imposes limits on sustained cortical acetylcholine release and attention. J Neurosci 33:2326-2337. CrossRef Medline

Robbins TW, Gillan CM, Smith DG, de Wit S, Ersche KD (2012) Neurocognitive endophenotypes of impulsivity and compulsivity: towards dimensional psychiatry. Trends Cogn Sci 16:81-91. CrossRef Medline

Robinson TE, Berridge KC (1993) The neural basis of drug craving: an incentive-sensitization theory of addiction. Brain Res Rev 18:247-291. CrossRef Medline

Robinson TE, Flagel SB (2009) Dissociating the predictive and incentive motivational properties of reward-related cues through the study of individual differences. Biol Psychiat 65:869-873. CrossRef Medline

Rogers RD, Everitt BJ, Baldacchino A, Blackshaw AJ, Swainson R, Wynne K, Baker NB, Hunter J, Carthy T, Booker E, London M, Deakin JF, Sahakian BJ, Robbins TW (1999) Dissociable deficits in the decision-making cognition of chronic amphetamine abusers, opiate abusers, patients with focal damage to prefrontal cortex, and tryptophan-depleted normal volunteers: evidence for monoaminergic mechanisms. Neuropsychopharmacology 20:322-339. CrossRef Medline

Rueter LE, Anderson DJ, Briggs CA, Donnelly-Roberts DL, Gintant GA, Gopalakrishnan M, Lin NH, Osinski MA, Reinhart GA, Buckley MJ, Martin RL, McDermott JS, Preusser LC, Seifert TR, Su Z, Cox BF, Decker MW, Sullivan JP (2004) ABT-089: pharmacological properties of a neuronal nicotinic acetylcholine receptor agonist for the potential treatment of cognitive disorders. CNS Drug Rev 10:167-182. Medline

Sarter M, Paolone G (2011) Deficits in attentional control: cholinergic mechanisms and circuitry-based treatment approaches. Behav Neurosci 125:825-835. CrossRef Medline

Sarter M, Gehring WJ, Kozak R (2006) More attention must be paid: the neurobiology of attentional effort. Brain Res Rev 51:145-160. CrossRef Medline 
Sarter M, Bruno JP, Parikh V (2007) Abnormal neurotransmitter release underlying behavioral and cognitive disorders: toward concepts of dynamic and function-specific dysregulation. Neuropsychopharmacology 32:1452-1461. CrossRef Medline

Saunders BT, Robinson TE (2010) A cocaine cue acts as an incentive stimulus in some but not others: implications for addiction. Biol Psychiatry 67:730-736. CrossRef Medline

Saunders BT, Robinson TE (2012) The role of dopamine in the accumbens core in the expression of Pavlovian-conditioned responses. Eur J Neurosci 36(4):2521-2532. CrossRef Medline

St Onge R, Stopper CM, Zahm DS, Floresco SB (2012) Separate prefrontalsubcortical circuits mediate different components of risk-based decision making. J Neurosci 32:2886-2899. CrossRef Medline

St Peters M, Cherian AK, Bradshaw M, Sarter M (2011a) Sustained attention in mice: expanding the translational utility of the SAT by incorporating the Michigan controlled access response port (MICARP). Behav Brain Res 225:574-583. CrossRef Medline

St Peters M, Demeter E, Lustig C, Bruno JP, Sarter M (2011b) Enhanced control of attention by stimulating mesolimbic-corticopetal cholinergic circuitry. J Neurosci 31:9760-9771. CrossRef Medline
Sullivan JP, Donnelly-Roberts D, Briggs CA, Anderson DJ, Gopalakrishnan M, Xue IC, Piattoni-Kaplan M, Molinari E, Campbell JE, McKenna DG, Gunn DE, Lin NH, Ryther KB, He Y, Holladay MW, Wonnacott S, Williams M, Arneric SP (1997) ABT-089 [2-methyl-3-(2-(S)-pyrrolidinylmethoxy)pyridine]: I. A potent and selective cholinergic channel modulator with neuroprotective properties. J Pharmacol Exp Ther 283: 235-246. Medline

Winstanley CA, Olausson P, Taylor JR, Jentsch JD (2010) Insight into the relationship between impulsivity and substance abuse from studies using animal models. Alcohol Clin Exp Res 34:1306-1318. CrossRef Medline

Xiao C, Nashmi R, McKinney S, Cai H, McIntosh JM, Lester HA (2009) Chronic nicotine selectively enhances $\alpha 4 \beta 2^{\star}$ nicotinic acetylcholine receptors in the nigrostriatal dopamine pathway. J Neurosci 29: 12428-12439. CrossRef Medline

Záborszky L, Cullinan WE (1992) Projections from the nucleus accumbens to cholinergic neurons of the ventral pallidum: a correlated light and electron microscopic double-immunolabeling study in rat. Brain Res 570: 92-101. CrossRef Medline 